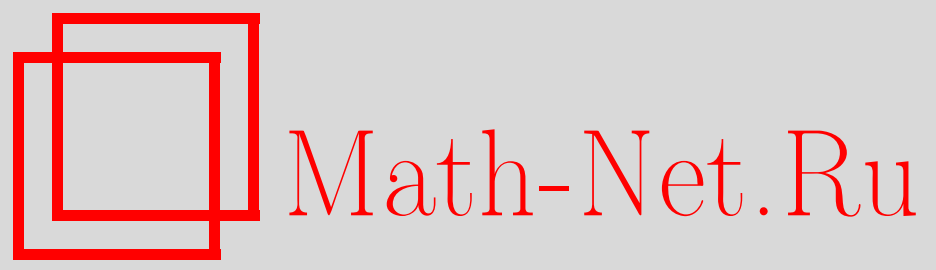

J.-Ch. Breton, Yu. A. Davydov, Local invariance principle for independent and identically distributed random variables, Теория вероятн. и ее примен., 2006, том 51, выпуск 2, 333-357

DOI: https://doi.org/10.4213/tvp57

Использование Общероссийского математического портала Math-Net.Ru подразумевает, что вы прочитали и согласны с пользовательским соглашением

http: //www . mathnet.ru/rus/agreement

Параметры загрузки:

IP : 3.80 .181 .102

26 апреля 2023 г., 15:50:20

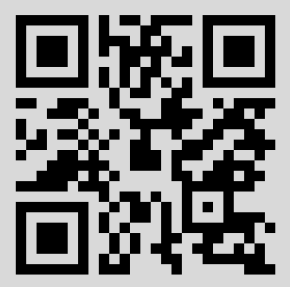




\title{
LOCAL INVARIANCE PRINCIPLE FOR INDEPENDENT AND IDENTICALLY DISTRIBUTED RANDOM VARIABLES
}

\begin{abstract}
Хорошо известно, что для последовательности независимых одинаково распределенных случайных величин соответствующие нормированные ступенчатые процессы слабо сходятся к винеровскому процессу. Более сильная сходимость, а именно, сходимость по вариации распределений функционалов от этих процессов, была установлена в [4] в предположении конечности информации Фишера для случайных величин. В настояшей статье мы доказываем такую сходимость без каких-либо условий, связанных с информацией Фишера.
\end{abstract}

Ключевые слова и фразы: принципы инвариантности, сходимость по вариации, локальные предельные теоремы.

\section{Introduction}

Let $\left\{\xi_{n}, n \in \mathbf{N}\right\}$ be a sequence of independent and identically distributed random variables defined on a probability space $(\Omega, \mathscr{F}, \mathbf{P})$. Let $\mathbf{E} \xi_{n}=0$ and $\mathbf{E} \xi_{n}^{2}=1$. Consider the following right-continuous process:

$$
S_{n}(t)=\frac{1}{\sqrt{n}} \sum_{i=1}^{[n t]} \xi_{i}, \quad t \in[0,1] .
$$

Let $P_{n}$ and $P$ stand for the laws of $S_{n}$ and the standard Wiener process $W$, respectively, in the Skorokhod space $\mathbb{D}$ of cadlag functions on the interval $[0,1]$. We use the symbol $\mu f^{-1}$ to denote the image of a measure $\mu$ on Borel $\sigma$-algebra $\mathcal{B}_{\mathbb{D}}$ under a measurable application $f: \mu f^{-1}(A)=\mu\left(f^{-1}(A)\right)$, $A \in \mathcal{B}_{\mathbb{D}}$. It is well known from the Donsker-Prokhorov invariance principle that, for any $P$-almost everywhere continuous functional $f: \mathbb{D} \rightarrow \mathbf{R}$,

$$
P_{n} f^{-1} \Longrightarrow P f^{-1}, \quad n \rightarrow \infty
$$

where $\Rightarrow$ denotes usual weak convergence in $\mathbf{R}$.

* Laboratoire de Mathématiques et Applications, Avenue Michel Crépeau, Université de La Rochelle, 17042 La Rochelle cedex; e-mail: jcbreton@univ-lr.fr

** Laboratoire P. Painlevé, U.M.R. CNRS 8524, Bât. M2, http://math.univ-lille1.fr, Cité Scientifique, 59655 Villeneuve d'Ascq Cedex, France; e-mail: youri.davydov@ math.univ-lille1.fr 
Our goal is to strengthen the result of (1.2) by proving strong convergence, in other words, convergence in variation of $P_{n} f^{-1}$ to $P f^{-1}$, for a large class of functionals $f$.

If the laws $P_{n} f^{-1}$ and $P f^{-1}$ are absolutely continuous with respect to the Lebesgue measure $\lambda$, then the strong convergence becomes equivalent to the convergence of densities $\frac{d P_{n} f^{-1}}{d \lambda}$ to $\frac{d P f^{-1}}{d \lambda}$ in the $L^{1}(\mathbf{R})$-metric. In this way, we get as a corollary the local limit theorem for the laws of functionals $f\left(S_{n}(\cdot)\right)$.

We note at the outset that if the measures $P_{n}$ themselves converge in variation to $P$, the answer to our question becomes obvious. This situation, however, rarely occurs in practice. In general, like in our case, the measures $P_{n}$ are singular with respect to $P$ for any $n$ and this mutual singularity of $P_{n}$ and $P$ is the cause of difficulties in solving the problem.

A first solution of the problem above is given in [4, Theorem 20.1] where the strong convergence

$$
P_{n} f^{-1} \stackrel{\text { varr }}{\longrightarrow} P f^{-1}
$$

is obtained assuming that the Fisher information $I_{p}=\int_{\mathbf{R}} p^{-1}\left(p^{\prime}\right)^{2} d \lambda$ of the density $p$ of $\xi_{1}$ is finite, furthermore, assuming that the function $f$ is an element of a certain class of functions, class denoted by $\mathscr{M}_{P}$. More precisely, the following theorem holds.

Theorem A. Let $\left\{\xi_{n}, n \in \mathbf{N}\right\}$ be a sequence of independent and identically distributed random variables with $\mathbf{E} \xi_{n}=0, \mathbf{E} \xi_{n}^{2}=1$. Suppose that their common law $F$ has an absolutely continuous density $p$ with $I_{p}<\infty$, then we have (1.3) for any functional $f$ belonging to the class $\mathscr{M}_{P}$.

We only note here that the class $\mathscr{M}_{P}$ is completely defined by the measure $P$, and for its definition and properties, we refer the reader to $[4, \mathrm{Sec}-$ tion 19].

The condition $I_{p}<\infty$, which is equivalent to $p^{\prime} / p \in L^{2}(d F)$, seems to be unnecessarily strong. Indeed it is more restrictive than $p^{\prime} / p \in L^{1}(d F)$ which means $\int\left|p^{\prime}\right| d \lambda<\infty$, that is, the bounded variation of $p$, whereas it is well known that for simple functionals such as $x \mapsto \sup _{t} x(t)$, the condition $F \ll \lambda$ is sufficient to guarantee a local limit theorem (see [5]).

The goal of this work is to weaken the main condition $I_{p}<\infty$.

We achieve this goal by narrowing slightly the class $\mathscr{M}_{P}$ to a certain one denoted by $\mathscr{M}_{P}^{(1)}$ and rigorously defined in Definition 1 below. First we give some additional notation. Since $\mathbb{D}$ is not separable when equipped with the uniform topology, we introduce its subspace $\mathbb{E}$ which is the uniform closure of the set of all functions of $\mathbb{D}$ that have finitely many jumps at rational points of $[0,1]$. When equipped with the uniform norm, $\mathbb{E}$ is a separable Banach space in which the weak convergence (1.2) still holds. We recall that $H_{P}$ stands for the core of the measure $P$. That is, $H_{P}$ is the set of admissible directions $l \in \mathbb{E}$ for $P\left(P T_{l}^{-1} \ll P\right.$, where $T_{l}$ is the translation of $\left.l\right)$ which 
coincides with the Cameron-Martin space: $H_{P}=\{f \in C([0,1]) \mid f(0)=0$, $\left.f^{\prime} \in L^{2}([0,1])\right\}$. Let us denote by $S$ the unit ball of $\mathbb{E}$.

D ef in ition $1 . \mathscr{M}_{P}^{(1)}$ is the set of locally Lipschitzian functionals $f$ such that for $P$-almost all $x$, there are a neighborhood $V(x)$ of $x$ and $l \in H_{P}$ such that

(a) the derivative $D_{l} f(x)$ of $f$ at $x$ in the direction $l$ exists and is not zero;

(b) denoting $S_{y}=\left\{h \in S \mid D_{h} f(y)\right.$ exists $\}$ and $A=\bigcup_{y \in V(x)}\{y\} \times S_{y}$, we have $A \ni(y, h) \rightarrow D_{h} f(y)$ bounded and continuous.

With $t_{-}=\sup \{t \in \mathbf{R} \mid F(t)=0\}, t_{+}=\inf \{t \in \mathbf{R} \mid F(t)=1\}$, where as usual, $\sup \varnothing=-\infty$ and $\inf \varnothing=+\infty$, our main result states the following.

Theorem 1. Let $\left\{\xi_{n}, n \in \mathbf{N}\right\}$ be a sequence of independent and identically distributed random variables with mean 0 and variance 1 . Suppose there exists some $\gamma>0$ such that $\mathbf{E}\left|\xi_{1}\right|^{2+\gamma}<\infty$. Furthermore, suppose $\xi_{1}$ has a density denoted by $p$, which is nonzero almost everywhere on $\left[t_{-}, t_{+}\right]$. Then the convergence (1.3) holds for any functional $f \in \mathscr{M}_{P}^{(1)}$.

In comparison with the quoted result of [4], in Theorem 1 we have substantially weakened the hypothesis on the law $F$. The price we have paid for this does not seem to be high: existence of an absolute moment greater than 2 and a slight restriction of the functional class. This restriction on the class can only be noticed on a formal level, since concrete functionals belong to the class $\mathscr{M}_{P}^{(1)}$ under hypothesis near to those used for $\mathscr{M}_{P}$. For example, our class contains

(a) all not degenerated linear continuous functionals;

(b) all differentiable functionals $f$ with $D f(x) \neq 0 P$-a.e.

The functionals of supremum type or integral type belong to the class $\mathscr{M}_{P}^{(1)}$ under hypothesis given below.

Proposition 1. The following functionals belong to $\mathscr{M}_{P}^{(1)}$ :

1) Let

$$
g(x)=\sup _{t \in[0,1]} \varphi(x(t)),
$$

where $\varphi$ is convex, $C^{1}$ and such that $\varphi^{\prime} \neq 0$ a.e.;

2) Let

$$
h(x)=\int_{0}^{1} q(x(t)) d t,
$$

where $q$ is $C^{1}$ and such that $q^{\prime}$ does not vanish a.e. in some neighborhood of 0 .

$\mathrm{R} \mathrm{e} \mathrm{m}$ a r k 1 . The conditions in the preceding examples can be weakened, relaxing the condition $\varphi$ of class $C^{1}$ in the first case and replacing, in the second, $q$ of class $C^{1}$ by $q$ Lipschitzian on all compacts. In this way, $g$ and $h$ may be not in $\mathscr{M}_{P}^{(1)}$, but the conclusion (1.3) of Theorem 1 still holds since the proof can be carried out in a similar way (see Remarks 5,6 
in Section 4). On the other hand, we cannot weaken the convexity condition for $\varphi$ because the convexity of $g$ is essential for the proof.

We have the following proposition concerning functionals in $\mathscr{M}_{P}^{(1)}$.

Proposition 2. For $f \in \mathscr{M}_{P}^{(1)}$, we have $P f^{-1} \ll \lambda$.

Proposition 2 was proved in [4] for functionals in a subclass of $\mathscr{M}_{P}$ which is larger than $\mathscr{M}_{P}^{(1)}$. This proposition justifies we can derive local limit theorem from Theorem 1.

$\mathrm{R} \mathrm{e} \mathrm{m} \mathrm{a} \mathrm{r} \mathrm{k}$ 2. In the case of the functional $f(x)=x(1)$, Theorem 1 and Proposition 2 yield a $L^{1}$-local limit theorem for the densities of partial sums of normalized random variables $\left(\xi_{i}\right)_{i}$. In comparison with the classical theorem by Yu. Prokhorov (see [6, Theorem 4.4.1]) our conditions on $F$ are more restrictive. However, it is not surprising since we are working with a very large class of functionals.

The proof of Theorem 1 relies on the so-called superstructure method which was introduced and used in [4]. Namely, we apply a partition of the space to express the functional distributions as mixtures of conditional distributions. The main newness of the proof of the current paper consists in representing initial random variables $\xi_{n}$ as transformed orthogaussian random variables $\eta_{n}: \xi_{n}=U\left(\eta_{n}\right)$, and then considering the process $S_{n}(t)$ as function of analogous process $Z_{n}(t)$ defined by $\left(\eta_{n}\right)_{n \geqslant 1}$. This implies that in place of admissible translations of $S_{n}(t)$ used in the proof of Theorem A, we use now nonlinear transformations induced by admissible translations of $W$. The analysis of the asymptotic behavior of the corresponding conditional law becomes more intricate and requires supplementary tools.

Remark also that the proof developed in the current paper can easily be adapted so that Theorem 1 would still hold for $P_{n}^{\prime}$, the laws of polygonal process $S_{n}^{\prime}$ defined by

$$
S_{n}^{\prime}(t)=\frac{1}{\sqrt{n}} \sum_{i=1}^{[n t]} \xi_{i}+\frac{n t-[n t]}{\sqrt{n}} \xi_{[n t]+1}
$$

(compare (1.6) to (1.1)).

The paper is organized as follows. We begin by specifying in Section 2 the notation and tools we use. We prove Theorem 1 in Section 3. Examples of functionals in $\mathscr{M}_{P}^{(1)}$ are discussed in Section 4. Some technical and tedious considerations are deferred to appendices.

\section{Preliminary tools and the main idea}

Let $\mathbb{E}_{n}=\{x \in \mathbb{E} \mid x$ is constant on $[k / n,(k+1) / n), k=0,1, \ldots, n-1\}$ and define the canonical surjection $\Pi_{n}: \mathbb{E} \rightarrow \mathbb{E}_{n}$ given by

$$
\Pi_{n}(x)(t)=\sum_{k=0}^{[n t]} x\left(\frac{k}{n}\right) \mathbf{1}_{[k / n,(k+1) / n)}+x(1) \mathbf{1}_{\{1\}}
$$


and the isomorphism $J_{n}: \mathbb{E}_{n} \rightarrow \mathbf{R}^{n}$ given by

$$
J_{n}(x)_{k}=\sqrt{n}\left(x\left(\frac{k}{n}\right)-x\left(\frac{k-1}{n}\right)\right) .
$$

Denote by $F$ the distribution function of $\xi_{1}$ and let $F^{-1}(y)=$ $\inf \{x \mid F(x) \geqslant y\}$ be its inverse; let $\Phi$ be the distribution function of the standard Gaussian law, $\lambda_{[a, b]}$ the Lebesgue measure on $[a, b]$ and $\bar{\lambda}$ the normalized Lebesgue measure on a finite interval. Finally, $C$ is a universal constant which may differ from place to place.

The proof of Theorem 1 consists in the analysis and verification of the conditions of the following superstructure result.

Theorem B (see [4, Theorem 18.4]). Consider a sequence of probability measures $\left\{P_{n}, n \in \overline{\mathrm{N}}\right\}$ defined on the Borel $\sigma$-algebra $\mathscr{B}_{\mathscr{X}}$ of a complete separable metric space $(\mathscr{X}, d)$. Suppose that $P_{n} \Rightarrow P_{\infty}$. Furthermore, suppose that, for $P_{\infty}$-almost all $x$, there exist an open ball $V$ centered at $x, a$ number $\varepsilon>0$, and also a family $\left(G_{n, c}, n \in \overline{\mathbf{N}}, c \in(0, \varepsilon]\right)$ of measurable transformations of $\mathscr{X}$ such that the following five conditions are satisfied:

(i) for each $c \in(0, \varepsilon)$ and each $\delta>0, \lim _{n \rightarrow \infty} P_{n}\left\{x \mid d\left(G_{n, c} x-G_{\infty, c} x\right) \geqslant\right.$ $\delta\}=0$;

(ii) for each $c \in(0, \varepsilon)$, the mapping $G_{\infty, c}$ is $P_{\infty}$-a.e. continuous; moreover, $\rho(S, c)=\sup _{z \in S} d\left(z, G_{\infty, c} z\right) \rightarrow 0$ when $c \rightarrow 0$, for each open ball $S$;

(iii) $\lim _{c \rightarrow 0} \varlimsup_{n \rightarrow \infty}\left\|P_{n} G_{n, c}^{-1}-P_{n}\right\|=0$;

(iv) for each $\delta \in(0, \varepsilon)$, suppose that

$$
\int_{V}\left\|\lambda_{[0, \delta]} \varphi_{n, z}^{-1}-\lambda_{[0, \delta]} \varphi_{\infty, z}^{-1}\right\| P_{n}(d z) \longrightarrow 0 \quad \text { as } n \rightarrow \infty
$$

where $\varphi_{n, z}(c)=f\left(G_{n, c} z\right)$ with $n \in \overline{\mathbf{N}}$ and $c \in(0, \varepsilon]$;

(v) for each $\delta \in(0, \varepsilon)$, the mapping $z \longmapsto \lambda_{[0, \delta]} \varphi_{\infty, z}^{-1}$ of $V$ into $\mathscr{Z}(\mathbf{R})$, the Banach space of signed measure on $\mathbf{R}$ normed with the total variation, is $P_{\infty}$-a.e. continuous.

Then.

$$
P_{n} f^{-1} \stackrel{\text { var }}{\longrightarrow} P_{\infty} f^{-1} \text {. }
$$

Theorem B (for proof see [4, Section 18]) needs some explanations. Therefore, we shall now quickly go through the notation of Theorem B in the set-up of Section 1 above, that is, in the case when $P_{n}$ is the probability law of the process $S_{n}$ of (1.1) in the space $\mathbb{E}$.

For $x$ in the $P$-full set occurring in Definition 1 of $\mathscr{M}_{P}^{(1)}$, let $V_{1}(x)=$ $B\left(x, r_{1}\right)$ be an open ball and let $l$ be a direction.

Since $D_{l} f(x) \neq 0$ by the definition of $\mathscr{M}_{P}^{(1)}$, we can suppose $D_{l} f(x)>0$ even if we change $l$ into $-l$. Using continuity of $D . f(\cdot)$ at $\left(x, l\|l\|^{-1}\right) \in A$, we get a neighborhood $B\left(x, r_{2}\right) \times B\left(l\|l\|^{-1}, r_{2}^{\prime}\right) \cap A$ with the property $B\left(x, r_{2}\right) \subset$ 
$B\left(x, r_{1}\right)$ and such that $f$ is Lipschitzian on $B\left(x, r_{2}\right)$ and furthermore for every $(y, h)$ in this neighborhood of $\left(x, l\|l\|^{-1}\right)$ we have

$$
D_{h} f(y) \geqslant \frac{1}{2} D_{l /\|l\| \|} f(x)>0 .
$$

In what follows, we consider the open ball $V(x)=B\left(x, r_{3}\right)$ with $r_{3} \leqslant r_{2} / 5$ and $\varepsilon<r_{2} / a\|l\|$. Assume $P(\partial V(x))=0$ (reducing $V(x)$ if it is not the case).

Let $U_{n}=F\left(\xi_{n}\right)$ (so that $U_{n}$ are uniform i.i.d. random variables), then $\eta_{n}=\Phi^{-1}\left(U_{n}\right)$ are orthogaussian variables and we have the obvious but useful representation $\xi_{n}=F^{-1} \circ \Phi\left(\eta_{n}\right)$.

Define

$$
U(x)=F^{-1} \circ \Phi(x) \text { and } V(x)=\Phi^{-1} \circ F(x) .
$$

Furthermore, define for $\bar{y}=\left(y_{1}, \ldots, y_{n}\right), \bar{x}=\left(x_{1}, \ldots, x_{n}\right)$

$\varphi(\bar{y})=\left(V\left(y_{1}\right), \ldots, V\left(y_{n}\right)\right) ; \quad \psi(\bar{x})=\left(U\left(x_{1}\right), \ldots, U\left(x_{n}\right)\right) ; \quad \bar{G}_{n, c}(\bar{x})=\bar{x}+c \bar{l}_{n}$, where $\bar{l}_{n}=J_{n} \circ \Pi_{n}(l)=\left(l_{n, i}\right)_{i \leqslant n}$ with

$$
l_{n, i}=\sqrt{n}\left(l\left(\frac{i}{n}\right)-l\left(\frac{i-1}{n}\right)\right)
$$

and $l \in H_{P}$ given by the definition of $\mathscr{M}_{P}^{(1)}$.

The following commutative diagram sums up the situation:

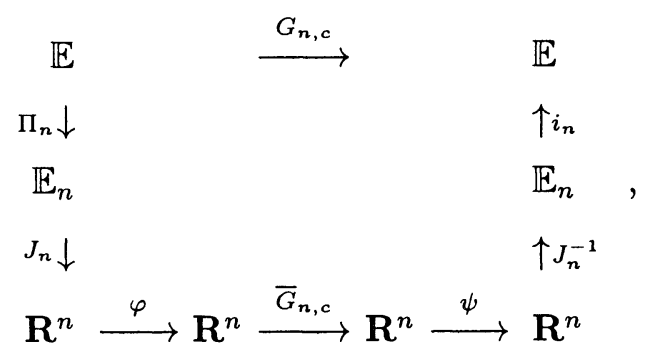

where, for each $n \in \overline{\mathbf{N}}$, the transformation $G_{n, c}$ is given by

$$
G_{n, c}=i_{n} \circ J_{n}^{-1} \circ \psi \circ \bar{G}_{n, c} \circ \varphi \circ J_{n} \circ \Pi_{n}, \quad G_{\infty, c}=x+c a l,
$$

$a$ in (2.3) is defined as follows:

$$
a=\int U^{\prime}(x) q(x) d x .
$$

R e m a r k 3. (i) Using the above definitions of $G_{n, c}$, we can obtain the following explicit expression:

$$
G_{n, c} x(t)=\sum_{k \leqslant[n t]} \frac{U\left(V(\sqrt{n}(x(k / n)-x((k-1) / n)))+c l_{n, k}\right)}{\sqrt{n}} .
$$


(ii) The function $U=F^{-1} \circ \Phi$ is absolutely continuous on any finite interval $[a, b]$. Indeed, since the function $F$ has a derivative $p>0$ a.e., it is easy to see from $\left[4\right.$, Theorem 4.2] that $\lambda_{[a, b]} F^{-1} \ll \lambda$. From the latter fact, we easily infer that $F^{-1}$ is absolutely continuous on $[F(a), F(b)]$. Consequently, the absolute continuity of $U$ on any finite intervals follows.

In particular, $U$ is differentiable a.e. and for any $d U$-integrable function $f$, we easily show that

$$
\int f(t) U^{\prime}(t) d t=\int f(t) d U(t)
$$

(iii) The quantity $a$ is well defined in (2.4) since we can see the convergence of the integral by using, for example, the following considerations:

$$
\int_{0}^{\infty} U^{\prime}(x) q(x) d x \leqslant \sum_{n=0}^{\infty} q(n)(U(n+1)-U(n)) \leqslant \sum_{n=0}^{\infty} q(n) U(n+1) .
$$

Since $\xi_{1} \in L^{2}(\Omega, \mathscr{F}, \mathbf{P})$, statement (A.1) in Appendix A.1 ensures the convergence of the sum on the right-hand side of (2.5).

(iv) Since $l$ is an element of the Cameron-Martin space $H_{P}$, we obtain the following:

$$
\begin{aligned}
\sum_{i=1}^{n} l_{n, i}^{2} & =n \sum_{i=1}^{n}\left(l\left(\frac{i}{n}\right)-l\left(\frac{i-1}{n}\right)\right)^{2}=\frac{1}{n} \sum_{i=1}^{n}\left(n \int_{(i-1) / n}^{i / n} l^{\prime}(s) d s\right)^{2} \\
& \leqslant \sum_{i=1}^{n} \int_{(i-1) / n}^{i / n} l^{\prime}(s)^{2} d s=\left\|l^{\prime}\right\|_{2}^{2} ; \\
\frac{1}{\sqrt{n}} \sum_{i=1}^{n}\left|l_{n, i}\right| & \leqslant \sum_{i=1}^{n}\left|l\left(\frac{i}{n}\right)-l\left(\frac{i-1}{n}\right)\right| \leqslant \sum_{i=1}^{n} \int_{(i-1) / n}^{i / n}\left|l^{\prime}(s)\right| d s \leqslant \int_{0}^{1}\left|l^{\prime}(s)\right| d s ; \\
\max _{i \leqslant n}\left|l_{n, i}\right| & =\sqrt{n}\left|\int_{(i-1) / n}^{i / n} l^{\prime}(s) d s\right| \leqslant\left(\int_{(i-1) / n}^{i / n} l^{\prime}(s)^{2} d s\right)^{1 / 2} \longrightarrow 0,
\end{aligned}
$$

where the convergence to 0 in $(2.8)$ holds because $l^{\prime} \in L^{2}([0,1])$. In what follows, we shall use $h$ to denote an upper bound of $\left\{l_{n, i}, i \leqslant n, n \in \mathbf{N}\right\}$.

(v) Since the result of Theorem 1 concerns the convergence of the probability laws $\left(P_{n}\right)_{n}$, there is no restriction to apply Skorokhod's representation theorem (see [1, Theorem 6.7]) and to suppose that we work with a probability space $(\widetilde{\Omega}, \widetilde{\mathscr{F}}, \widetilde{\mathbf{P}})$ and processes $\widetilde{S}_{n}, \widetilde{W}$ defined on this space such that for all $n \in \mathbf{N}$,

$$
\widetilde{S}_{n} \stackrel{\mathscr{L}}{=} S_{n}, \quad \widetilde{W} \stackrel{\mathscr{L}}{=} W, \quad \widetilde{S}_{n} \longrightarrow \widetilde{W} \quad \widetilde{\mathbf{P}}_{\text {-a.s. }}
$$

In the same way, we have $\widetilde{S}_{n}=n^{-1 / 2} \sum_{i \leqslant[n t]} \tilde{\xi}_{i}^{n}$ with independent and identically distributed random variables $\tilde{\xi}_{i}^{n} \stackrel{\mathscr{L}}{=} \xi_{i}$ having the density $p$; analogously, we have $\left(\tilde{\xi}_{i}^{n}\right)_{i \leqslant n}=\left(U\left(\tilde{\eta}_{i}^{n}\right)\right)_{i \leqslant n}$, where $\left(\tilde{\eta}_{i}^{n}\right)$ are independent $\mathscr{N}(0,1)$ random variables. 
In order to not complicate notation, we will keep on writing $S_{n}$ and $W$ for $\widetilde{S}_{n}, \widetilde{W}$ in the sequel, keeping in mind that for each $n$, the family $\left(\tilde{\xi}_{i}^{n}\right)_{i \leqslant n}$ changes.

\section{Proof}

The purpose is now to deal with conditions (i) $-(\mathrm{v})$ of Theorem B. The most difficult will be (i) and (iv).

3.1. Study of (i). In order to see, for any fixed $c \in(0, \varepsilon)$ and any $\alpha>0$, that

$$
P_{n}\left\{x|| G_{n, c} x-G_{\infty, c} x \mid \geqslant \alpha\right\}=\mathbf{P}\left\{\left\|G_{n, c} S_{n}-G_{\infty, c} S_{n}\right\| \geqslant \alpha\right\} \longrightarrow 0,
$$

as $n \rightarrow \infty$, remark first that we can write

$$
G_{n, c} S_{n}(\cdot)=\frac{1}{\sqrt{n}} \sum_{i \leqslant[n \cdot]} U\left(\eta_{i}^{n}+c l_{n, i}\right), \quad G_{\infty, c} S_{n}(\cdot)=\frac{1}{\sqrt{n}} \sum_{i \leqslant[n \cdot]} U\left(\eta_{i}^{n}\right)+c a l .
$$

Since $\|l([n \cdot] / n)-l(\cdot)\| \rightarrow 0$ as $n \rightarrow \infty$, we reduce the study to see the following:

$$
\left\|\frac{1}{\sqrt{n}} \sum_{i \leqslant[n \cdot]} U\left(\eta_{i}^{n}+c a l_{n, i}\right)-U\left(\eta_{i}^{n}\right)-c a l_{n, i}\right\| \stackrel{\mathbf{P}}{\longrightarrow} 0 .
$$

With

$$
\tau_{n, i}=U\left(\eta_{i}^{n}+c a l_{n, i}\right)-U\left(\eta_{i}^{n}\right)-c a l_{n, i}, \quad S_{n, k}=\sum_{i=1}^{k} \frac{\tau_{n, i}}{\sqrt{n}}
$$

we can write

$$
\left\|\frac{1}{\sqrt{n}} \sum_{i \leqslant[n \cdot]} U\left(\eta_{i}^{n}+c a l_{n, i}\right)-U\left(\eta_{i}^{n}\right)-\operatorname{cal}_{n, i}\right\|=\max _{k \leqslant n}\left|\frac{1}{\sqrt{n}} \sum_{i \leqslant k} \tau_{n, i}\right|=\max _{k \leqslant n}\left|S_{n, k}\right| .
$$

Since $\xi_{1} \in L^{2+\gamma}(\Omega, \mathscr{F}, \mathbf{P})$, we easily obtain by Hölder's inequality that

$$
\int U(x+h)^{2} q(x) d x<\infty
$$

and we deduce $\tau_{n, i} \in L^{2}(\Omega, \mathscr{F}, \mathbf{P})$.

We begin with the study of $\max _{k \leqslant n}\left|n^{-1 / 2} \sum_{i=1}^{k} \mathbf{E} \tau_{n, i}\right|$.

Using definition (2.4) of $a$, we have by Fubini's theorem

$$
\begin{aligned}
\mathbf{E} \tau_{n, i} & =\int\left(U\left(x+c l_{n, i}\right)-U(x)\right) q(x) d x-c a l_{n, i} \\
& =\int\left(\int_{\left[s-c l_{n, i}, s\right]}(q(x)-q(s)) d x\right) U^{\prime}(s) d s,
\end{aligned}
$$


where we have assumed $l_{n, i} \geqslant 0$ in order to write intervals $\left[x, x+c l_{n, i}\right]$ in an easier way (which imposes no restriction). Now we can derive

$$
\max _{k \leqslant n}\left|\frac{1}{\sqrt{n}} \sum_{i=1}^{k} \mathbf{E} \tau_{n, i}\right| \leqslant \frac{1}{\sqrt{n}} \sum_{i=1}^{n} \int\left|\int_{\left[s-c l_{n, i}, s\right]}(q(x)-q(s)) d x\right| U^{\prime}(s) d s .
$$

Since by (2.4), (3.4) integrals $\int U^{\prime}(s) e^{-s^{2} / 2} d s, \int U^{\prime}(s) e^{-(|s|-\varepsilon h)^{2} / 2} d s$ are convergent and $h$ is an upper bound of $\left\{l_{n, i}, i \leqslant n, n \in \mathbf{N}\right\}$, for any arbitrary fixed $\alpha>0$, we can choose $M \geqslant \varepsilon h$ such that

$$
\int_{|s| \geqslant M} U^{\prime}(s) e^{-s^{2} / 2} d s, \quad \int_{|s| \geqslant M} U^{\prime}(s) e^{-(|s|-\varepsilon h)^{2} / 2} d s \leqslant \frac{\alpha}{2 \varepsilon \int_{0}^{1}\left|l^{\prime}(s)\right| d s} .
$$

We then study the right-hand side of (3.5) splitting the outer integral in to two parts $\left(d_{1}\right)=\int_{|s|>M},\left(d_{2}\right)=\int_{|s| \leqslant M}$.

First,

$$
\left(d_{1}\right) \leqslant \int_{|s|>M}\left(\frac{1}{\sqrt{n}} \sum_{i=1}^{n}\left|\int_{\left[s-c l_{n, i}, s\right]} q(x) d x\right|+\frac{1}{\sqrt{n}} \sum_{i=1}^{n} c\left|l_{n, i}\right| q(s)\right) U^{\prime}(s) d s .
$$

Since $q(x)=e^{-x^{2} / 2} \leqslant e^{-(|s|-\varepsilon h)^{2} / 2}$ for $x \in\left[s-c l_{n, i}, s\right]$, we have

$$
\frac{1}{\sqrt{n}} \sum_{i=1}^{n}\left|\int_{\left[s-c l_{n, i}, s\right]} q(x) d x\right| \leqslant \varepsilon \int_{0}^{1}\left|l^{\prime}(s)\right| d s e^{-(|s|-\varepsilon h)^{2} / 2} .
$$

It follows with (2.7) and choice (3.6) of $M$ that $\left(d_{1}\right) \leqslant \alpha$.

Second, in order to study $\left(d_{2}\right)$, write

$$
\begin{aligned}
& \left|\int_{\left[s-c l_{n, i}, s\right]}(q(x)-q(s)) d x\right|=\left|e^{-s^{2} / 2} \int_{0}^{c l_{n, i}}\left(e^{s y-y^{2} / 2}-1\right) d y\right| \\
& \quad \leqslant K e^{-s^{2} / 2}\left|\int_{0}^{c l_{n, i}}\left(|s y|+\frac{y^{2}}{2}\right) d y\right| \leqslant K \frac{e^{-s^{2} / 2}}{2} \varepsilon^{2} l_{n, i}^{2}\left(|s|+\frac{\varepsilon h}{3}\right),
\end{aligned}
$$

where the last inequality is due to boundedness of exp by $K$ on a finite interval when $s \in[-M, M],|y| \leqslant \varepsilon h$. Since $|x| \leqslant M$, estimate (2.6) and choice (3.6) of $M$ ensure

$$
\left(d_{2}\right) \leqslant \frac{1}{\sqrt{n}} \frac{K \varepsilon^{2}}{2}\left(M+\frac{\varepsilon h}{3}\right)\left\|l^{\prime}\right\|_{2}^{2} \int e^{-s^{2} / 2} U^{\prime}(s) d s=O\left(\frac{1}{\sqrt{n}}\right) .
$$

From (3.5), (3.7), and $\left(d_{1}\right) \leqslant \alpha$, we deduce

$$
\varlimsup_{n} \max _{k \leqslant n}\left|\frac{1}{\sqrt{n}} \sum_{i=1}^{k} \mathbf{E} \tau_{n, i}\right|=0 .
$$

The study of (3.3) is thus brought back to

$$
\max _{k \leqslant n}\left|\sum_{i=1}^{k} \frac{\tau_{n, i}-\mathbf{E} \tau_{n, i}}{\sqrt{n}}\right| .
$$


Doob's maximal inequality, applied to the positive submartingale given by the absolute value of partial sums of independent centered variables, yields

$$
\begin{aligned}
& \mathbf{E}\left(\max _{k \leqslant n}\left|\sum_{i=1}^{k} \frac{\tau_{n, i}-\mathbf{E} \tau_{n, i}}{\sqrt{n}}\right|\right)^{2}=\mathbf{E} \max _{k \leqslant n}\left(\sum_{i=1}^{k} \frac{\tau_{n, i}-\mathbf{E} \tau_{n, i}}{\sqrt{n}}\right)^{2} \\
& \quad \leqslant 4 \mathbf{E}\left(\sum_{i=1}^{n} \frac{\tau_{n, i}-\mathbf{E} \tau_{n, i}}{\sqrt{n}}\right)^{2} \leqslant \frac{4}{n} \sum_{i=1}^{n} \mathbf{E}\left(U\left(\eta_{i}^{n}+c l_{n, i}\right)-U\left(\eta_{i}^{n}\right)\right)^{2} .
\end{aligned}
$$

Using Fubini's theorem, we have

$$
\begin{aligned}
& \mathbf{E}\left(U\left(\eta_{i}^{n}+c l_{n, i}\right)-U\left(\eta_{i}^{n}\right)\right)^{2} \\
& \quad=\int_{\mathbf{R}} U^{\prime}(s) \int_{\left[s-c l_{n, i}, s+c l_{n, i}\right]} U^{\prime}(t) \int \mathbf{1}_{I\left(s, t, c l_{n, i}\right)}(x) q(x) d x d t d s,
\end{aligned}
$$

where $I\left(s, t, c l_{n, i}\right)=\left[s-c l_{n, i}, s\right] \cap\left[t-c l_{n, i}, t\right]$ is of length smaller than $c\left|l_{n, i}\right|$. We split once more the outer integral into two parts:

$$
\left(d_{3}\right)=\int_{|s| \leqslant \varepsilon h}, \quad\left(d_{4}\right)=\int_{|s|>\varepsilon h} .
$$

Since $\int_{I\left(s, t, c l_{n, i}\right)} q(x) d x \leqslant c\left|l_{n, i}\right|$ and integrals over finite intervals are bounded, there is a constant $K_{3}<\infty$ such that

$$
\left(d_{3}\right) \leqslant K_{3} \varepsilon\left|l_{n, i}\right| .
$$

Since for $x \in I\left(s, t, c l_{n, i}\right)$, we have $|x| \geqslant|s|-\varepsilon h$, monotony of $q$ ensures

$$
\int_{I\left(s, t, c l_{n, i}\right)} q(x) d x \leqslant c\left|l_{n, i}\right| q(|s|-\varepsilon h) .
$$

Whence

$$
\begin{aligned}
\left(d_{4}\right) & \leqslant \int_{|s| \geqslant \varepsilon h}\left(U^{\prime}(s) c\left|l_{n, i}\right| q(|s|-\varepsilon h) \int_{\left[s-c l_{n, i}, s+c l_{n, i}\right]} U^{\prime}(t) d t\right) d s \\
& \leqslant \int_{|s| \geqslant \varepsilon h} U^{\prime}(s) c\left|l_{n, i}\right| q(|s|-\varepsilon h)(U(s+\varepsilon h)-U(s-\varepsilon h)) d s .
\end{aligned}
$$

Since from Appendix A.1: $U(x)=o\left((x / q(x))^{1 /(2+\gamma)}\right)$, we have for any fixed $\mu$ :

$$
q(|s|-\mu)\left(\frac{s+h}{q(s+h)}\right)^{1 /(2+\gamma)}=o\left(\exp \left\{-\frac{s^{2}}{16}\right\}\right) .
$$

Comparing $\int_{0}^{\infty} U^{\prime}(s) \exp \left\{-s^{2} / 16\right\} d s$ to a series, we obtain the convergence of $\int_{|s| \geqslant \mu} q(|s|-\mu) U(s+h) d U(s)$. We deduce from (3.13) that there is a constant $K_{4}<\infty$ such that

$$
\left(d_{4}\right) \leqslant K_{4} \varepsilon\left|l_{n, i}\right| .
$$


It follows from (3.10)-(3.12), (3.14), using (2.7):

$$
\mathbf{E}\left(\max _{k \leqslant n}\left|\sum_{i=1}^{k} \frac{\tau_{n, i}-\mathbf{E} \tau_{n, i}}{\sqrt{n}}\right|\right)^{2} \leqslant \frac{1}{\sqrt{n}}\left(K_{3}+K_{4}\right) \varepsilon \int_{0}^{1}\left|l^{\prime}(s)\right| d s .
$$

We thus obtain (3.3) from (3.8), (3.15). Finally, (3.1) is satisfied and so is point (i) of Theorem B.

3.2. Study of (ii). Mappings $G_{\infty, c}$ are obviously continuous, and using $d \rightarrow \infty$ for the uniform metric we get $d_{\infty}\left(z, G_{\infty, c} z\right)=c a\|l\| \rightarrow 0$ as $c \rightarrow 0$, so (ii) is easily justified.

3.3. Study of (iii). We aim to see

$$
\lim _{c \rightarrow 0} \varlimsup_{n}\left\|P_{n} G_{n, c}^{-1}-P_{n}\right\|=0 .
$$

For $\bar{\eta}^{n}=\left(\eta_{1}^{n}, \ldots, \eta_{n}^{n}\right)$ with law $\mathscr{P}_{n}$, we easily have

$$
S_{n}=\frac{1}{\sqrt{n}} \sum_{i=1}^{[n \cdot]} \xi_{i}^{n}=J_{n}^{-1}\left(\xi_{1}^{n}, \ldots, \xi_{n}^{n}\right)
$$

From notation (2.3), we have also $G_{n, c} S_{n}=J_{n}^{-1} \circ \psi\left(\bar{G}_{n, c} \bar{\eta}^{n}\right)$. It follows that

$$
\left\|P_{n} G_{n, c}^{-1}-P_{n}\right\| \leqslant\left\|\mathscr{P}_{n} \bar{G}_{n, c}^{-1}-\mathscr{P}_{n}\right\| \leqslant c\left\|l^{\prime}\right\|_{2},
$$

where the later inequality is due to Lemma 20.1 of [4] applied to the standard $n$-dimensional Gaussian law $\mathscr{P}_{n}$, and to the fact that the Fisher information of $\mathscr{N}(0,1)$ is 1 . Now (3.16) readily follows from (3.17).

3.4. Study of (v). In this section, we aim at seeing for any $\delta \in(0, \varepsilon)$ that for $P$-almost all $z \in V(x)$, the convergence $z_{n} \rightarrow z$ implies

$$
\left\|\lambda_{[0, \delta]} \varphi_{\infty, z_{n}}^{-1}-\lambda_{[0, \delta]} \varphi_{\infty, z}^{-1}\right\| \longrightarrow 0
$$

where we remind that $\varphi_{\infty, z}(c)=f\left(G_{\infty, c} z\right)$.

Let $\Delta_{n}=\left\{z_{n}+c a l, c \in[0, \delta]\right\}$ and $\Delta=\{z+c a l, c \in[0, \delta]\}$. Since $z_{n} \rightarrow z$, we have the convergence of segments $\Delta_{n} \rightarrow \Delta$.

Since $z_{n}, z \in B\left(x, r_{3}\right)$ and $c \leqslant \delta \leqslant \varepsilon \leqslant r_{3}(a\|l\|)^{-1}$, we have $\Delta_{n}, \Delta \subset$ $B\left(x, 2 r_{3}\right)$.

Choice of neighborhood ensures $f$ is Lipschitzian over $B\left(x, r_{2}\right)$ and $D \cdot f(\cdot)$ is continuous over $B\left(x, r_{2}\right) \times B\left(l\|l\|^{-1}, r_{2}^{\prime}\right) \cap A$. With $f_{\Delta_{n}}, f_{\Delta}$ standing for the restrictions of $f$ over segments $\Delta_{n}, \Delta$, we have

$$
\lambda_{[0, \delta]} \varphi_{\infty, z_{n}}^{-1}=\lambda f_{\Delta_{n}}^{-1}, \quad \lambda_{[0, \delta]} \varphi_{\infty, z}^{-1}=\lambda f_{\Delta}^{-1}
$$

and (3.18) is a consequence of the following proposition.

Proposition 3 (see [3, Corollary 2]). Let $g_{n}:[0,1] \rightarrow \mathbf{R}, n \in \overline{\mathbf{N}}$, be such that

1) $g_{n}$ is absolutely continuous for all $n \in \overline{\mathbf{N}}$;

2) $g_{n}(0) \rightarrow g_{\infty}(0)$ as $n \rightarrow \infty$; 
3) $g_{n}(1) \rightarrow g_{\infty}(1)$ as $n \rightarrow \infty$;

4) $g_{n}^{\prime}(c)>0$ a.e. for all $n \in \overline{\mathrm{N}}$

5) $g_{n}^{\prime}(c) \rightarrow g_{\infty}^{\prime}(c)$ a.e. as $n \rightarrow \infty$; then $\lambda g_{n}^{-1} \stackrel{\text { var }}{\rightarrow} \lambda g_{\infty}^{-1}$.

In our setting, points 1), 2), 3) are trivial. Point 4) follows from

$$
\left(z_{n}+c a l, l\|l\|^{-1}\right), \quad\left(z+c a l, l\|l\|^{-1}\right) \in B\left(x, r_{2}\right) \times B\left(l\|l\|^{-1}, r_{2}^{\prime}\right) \cap A,
$$

and (2.1). Point 5) is due to the continuity of $D \cdot f(\cdot)$ on $B\left(x, r_{2}\right) \times$ $B\left(l\|l\|^{-1}, r_{2}^{\prime}\right) \cap A$.

3.5. Study of (iv). We denote, for any $n \in \overline{\mathbf{N}}, \varphi_{n, z}: c \mapsto f\left(G_{n, c} z\right)$. We aim now at seeing that

$$
\varlimsup_{n} \int_{V}\left\|\lambda_{[0, \delta]} \varphi_{n, z}^{-1}-\lambda_{[0, \delta]} \varphi_{\infty, z}^{-1}\right\| P_{n}(d z)=0 .
$$

Write down for the sequel:

$$
\begin{array}{ll}
g_{n}(\omega, c)=f\left(G_{n, c} S_{n}\right), & g_{\infty}(\omega, c)=f\left(G_{\infty, c} W\right) ; \\
h_{n}(\omega, c)=f\left(G_{\infty, c} S_{n}\right), & h_{\infty}=g_{\infty} .
\end{array}
$$

Remind we work with a probability space due to Skorokhod's representation theorem [1, Theorem 6.7] for which (2.9) holds: $S_{n} \rightarrow W$ P-a.s.

3.6. Study of $\left(h_{n}\right)_{n \geqslant 1}$. The following proposition will be proved in Appendix A.3 using preceding Proposition 3.

Proposition 4. For $n \in \overline{\mathbf{N}}$, let $f_{n}:(\Omega \times[0, \delta], \mathscr{F} \times \mathscr{B}([0, \delta]), \mathbf{P} \otimes \bar{\lambda}) \rightarrow$ $\mathbf{R}, \Omega^{*} \in \mathscr{F}, \Omega^{*} \subset \Omega$ be such that

1) for all $\omega \in \Omega^{*}$, there exists $N_{1}(\omega)$, for all $n \geqslant N_{1}(\omega), f_{n}(\omega, \cdot)$ is absolutely continuous;

2) $f_{n}(\omega, 0) \stackrel{\mathbf{P}}{\longrightarrow} f_{\infty}(\omega, 0)$ on $\Omega^{*}$;

3) $f_{n}(\omega, \delta) \stackrel{\mathbf{P}}{\longrightarrow} f_{\infty}(\omega, \delta)$ on $\Omega^{*}$;

4) for all $\omega \in \Omega^{*}$ there exists $N_{4}(\omega)$ for all $n \geqslant N_{4}(\omega)$, we have $\frac{\partial}{\partial c} f_{n}(\omega, c)>0 \lambda$-a.e. for $c \in(0, \delta)$;

5) $\frac{\partial}{\partial c} f_{n}(\omega, c) \stackrel{\mathbf{P} \otimes \bar{\lambda}}{\longrightarrow} \frac{\partial}{\partial c} f_{\infty}(\omega, c)$ on $\Omega^{*}$.

Then $\left\|\lambda_{[0, \delta]} f_{n}(\omega, \cdot)^{-1}-\lambda_{[0, \delta]} f_{\infty}(\omega, \cdot)^{-1}\right\| \stackrel{\mathbf{P}}{\longrightarrow} 0$ on $\Omega^{*}$.

We apply now this proposition to the sequence $\left(h_{n}\right)_{n \geqslant 1}$.

(i) For $\omega \in W^{-1}(V)$ and $c \leqslant \delta \leqslant r_{3} / a\|l\|$, there is $N_{1}(\omega)$ such that for $n \geqslant N_{1}(\omega), S_{n}+$ cal $\in B\left(x, 3 r_{3}\right) \subset B\left(x, r_{2}\right)$, where $f$ is Lipschitzian. Required absolute continuity in point 1$)$ is easily derived.

(ii) We have for any $c \in[0, \delta], S_{n}+c a l \rightarrow W+c a l \in B\left(x, r_{2}\right)$ a.s. for $\omega \in W^{-1}(V)$. Continuity of $f$ ensures the a.s. convergence and thus convergence in probability of $f\left(S_{n}+c a l\right)$ to $f(W+c a l)$. Points 2$\left.), 3\right)$ are thus satisfied. 
(iii) For $n \geqslant N_{1}(\omega)$ and $c \leqslant \delta \leqslant r_{3} / a\|l\|$, we have $S_{n}+c a l \in B\left(x, r_{2}\right)$. It follows easily that ' $h_{n}(\omega, \cdot)$ is differentiable a.e. on $[0, \delta]$ and

$$
\frac{\partial}{\partial c} h_{n}(\omega, c)=D_{a l} f\left(G_{\infty, c} S_{n}\right) \text {. }
$$

Since $\left(S_{n}+c a l, l\|l\|^{-1}\right) \in B\left(x, r_{2}\right) \times B\left(l\|l\|^{-1}, r_{2}^{\prime}\right) \cap A$, we have for all $\omega \in W^{-1}(V), \frac{\partial}{\partial c} h_{n}(\omega, c)>0$ a.e. Similarly $\frac{\partial}{\partial c} h_{\infty}(\omega, c)>0$ and point 4$)$ is guaranteed.

(iv) Since $S_{n}+$ cal $\rightarrow$. $W+$ cal $\mathbf{P}$-a.s. and $\left(S_{n}+c a l, l\|l\|^{-1}\right) \in B\left(x, r_{2}\right) \times$ $B\left(l\|l\|^{-1}, r_{2}^{\prime}\right) \cap A$, where $D \cdot f(\cdot)$ is continuous, point 5$)$ is clear.

Proposition 4 applied to $\left(h_{n}\right)_{n \geqslant 1}$ yields for any $\alpha>0$,

$$
\lim _{n \rightarrow \infty} \mathbf{P}\left\{\omega \in W^{-1}(V),\left\|\lambda_{[0, \delta]} h_{n}(\omega, \cdot)^{-1}-\lambda_{[0, \delta]} h_{\infty}(\omega, \cdot)^{-1}\right\|>\alpha\right\}=0 .
$$

3.7. Study of $\left(g_{n}\right)_{n \geqslant 1}$. For $\left(g_{n}\right)_{n \geqslant 1}$, we use another version of Proposition 4 derived in the same way from a corollary of Proposition 3 (see Appendix A.3).

Proposition 5. For $n \in \overline{\mathbf{N}}$, let $f_{n}:(\Omega \times[0, \delta], \mathscr{F} \times \mathscr{B}([0, \delta])$, $\mathbf{P} \otimes \bar{\lambda}) \rightarrow \mathbf{R}, \Omega^{*} \in \mathscr{F}, \Omega^{*} \subset \Omega$ be such that

1) for all $\omega \in \Omega^{*}$, there exists $N_{1}(\omega)$, for all $n \geqslant N_{1}(\omega), f_{n}(\omega, \cdot)$ is absolutely continuous;

2) $f_{n}(\omega, 0) \stackrel{\mathbf{P}}{\longrightarrow} f_{\infty}(\omega, 0)$ on $\Omega^{*}$

3) for all $\omega \in \Omega^{*}$, we have $\frac{\partial}{\partial c} f_{\infty}(\omega, c)>0 \lambda$-a.e. for $c \in(0, \delta)$;

4) $\left\|\frac{\partial}{\partial c} f_{n}(\omega, c)-\frac{\partial}{\partial c} f_{\infty}(\omega, c)\right\|_{L^{1}} \stackrel{\mathbf{P}}{\longrightarrow} 0$ on $\Omega^{*}$.

Then $\left\|\lambda_{[0, \delta]} f_{n}(\omega, \cdot)^{-1}-\lambda_{[0, \delta]} f_{\infty}(\omega, \cdot)^{-1}\right\| \stackrel{\mathbf{P}}{\longrightarrow} 0$ on $\Omega^{*}$.

Verification of the hypotheses of this proposition for $\left(g_{n}\right)_{n \geqslant 1}$ requires a preliminary study of a tangent vector $L_{n, S_{n}, c}$ to the trajectory $\left(G_{n, c} S_{n}\right)_{c}$.

3.7.1. Study of the tangent vector. Using (3.2) and a.e. differentiability of $U$, we have, for each $t \in[0,1]$, for fixed $\omega \in \Omega, n \in \mathbf{N}$

$$
\frac{\partial}{\partial c}\left(G_{n, c} S_{n}(t)\right)=\frac{1}{\sqrt{n}} \sum_{i=1}^{[n t]} l_{n, i} U^{\prime}\left(\eta_{i}^{n}+c l_{n, i}\right) \text { a.e. }
$$

Let us show that $(3.22)$ holds in $\mathbb{E}$ for almost all $c \in[0, \delta]$. Since $\left|l_{n, i}\right| \leqslant h$, for $n$ fixed and almost all $\omega$ the differentiability of $U$ implies that for any $\varepsilon>0$ and almost all $c$, there is $\alpha(\omega, n, c)$ such that for $\delta \leqslant \alpha(\omega, n, c) / h$, $i=1, \ldots, n$ :

$$
\left|\frac{U\left(\eta_{i}^{n}+(c+\delta) l_{n, i}\right)-U\left(\eta_{i}^{n}+c l_{n, i}\right)-\delta l_{n, i} U^{\prime}\left(\eta_{i}^{n}+c l_{n, i}\right)}{\delta l_{n, i}}\right| \leqslant \varepsilon .
$$

We easily derive the following inequality using again (2.7):

$$
\left\|G_{n, c+\delta} S_{n}-G_{n, c} S_{n}-\frac{\delta}{\sqrt{n}} \sum_{i=1}^{[n]} l_{n, i} U^{\prime}\left(\eta_{i}^{n}+c l_{n, i}\right)\right\| \leqslant \varepsilon \delta \int_{0}^{1}\left|l^{\prime}(s)\right| d s .
$$


Finally, for all $\omega \in \Omega$, there is a set $A(\omega) \in \mathscr{B}([0, \delta]), \lambda(A(\omega))=0$ such that for all $n \in \mathbf{N}$ and $c \notin A(w)$, we have the differentiability of $G_{n, c} S_{n}$ at $c$ :

$$
L_{n, S_{n}, c}:=\frac{\partial}{\partial c}\left(G_{n, c} S_{n}\right)(c)=\frac{1}{\sqrt{n}} \sum_{i=1}^{[n]} l_{n, i} U^{\prime}\left(\eta_{i}^{n}+c l_{n, i}\right) .
$$

For $n=\infty$, the tangent vector is obviously al.

In the sequel, we need the convergence of $L_{n, S_{n}, c}$ to $a l$ in the following sense:

$$
\mathbf{E}\left(\frac{1}{\delta} \int_{0}^{\delta}\left\|L_{n, S_{n}, c}-a l\right\| d c\right) \longrightarrow 0, \quad n \rightarrow \infty .
$$

Expression (3.23) for $L_{n, S_{n}, c}$ and the convergence $\|l([n] / n)-l\| \rightarrow 0$ as $n \rightarrow \infty$ allow us to reduce the study to that of

$$
\left\|\frac{1}{\sqrt{n}} \sum_{i=1}^{[n]} l_{n, i}\left(U^{\prime}\left(\eta_{i}^{n}+c l_{n, i}\right)-a\right)\right\|=\max _{k \leqslant n}\left|\frac{1}{\sqrt{n}} \sum_{i=1}^{k} l_{n, i}\left(U^{\prime}\left(\eta_{i}^{n}+c l_{n, i}\right)-a\right)\right| .
$$

To this end, we use the following additional notation:

$$
\begin{gathered}
\hat{l}_{n, i}=\frac{l_{n, i}}{\sqrt{n}} ; \quad h(x)=U^{\prime}(x)-a ; \quad Z_{n}(c)=\sum_{i=1}^{[n]} \hat{l}_{n, i}\left(U^{\prime}\left(\eta_{i}^{n}+c l_{n, i}\right)-a\right) ; \\
\zeta_{n, i}(c)=h\left(\eta_{i}^{n}+c l_{n, i}\right) ; \quad \zeta_{n, i}^{s}(c)=\zeta_{n, i}(c) \mathbf{1}_{\left|\zeta_{n, i}(c)\right| \leqslant s}, \quad s>0 .
\end{gathered}
$$

Note that for $\eta$ an $\mathscr{N}(0,1)$ random variable, we have $\mathbf{E}|h(\eta)|<\infty$, $\mathrm{E} h(\eta)=0$ and integrability of $\zeta_{n, i}(c)$ follows from (3.4). Split $Z_{n}(c)$ as follows:

$$
Z_{n}(c)=\left(d_{5}\right)+\left(d_{6}\right)+\left(d_{7}\right)
$$

where

$$
\begin{gathered}
\left(d_{5}\right)=\sum_{i=1}^{[n]} \hat{l}_{n, i}\left(\zeta_{n, i}^{s}(c)-\mathbf{E} \zeta_{n, i}^{s}(c)\right), \quad\left(d_{6}\right)=\sum_{i=1}^{[n]} \hat{l}_{n, i} \mathbf{E} \zeta_{n, i}^{s}(c), \\
\left(d_{7}\right)=\sum_{i=1}^{[n]} \hat{l}_{n, i} \zeta_{n, i}(c) \mathbf{1}_{\left|\zeta_{n, i}(c)\right|>s} .
\end{gathered}
$$

In order to deal with $\left(d_{5}\right)$, we use Fubini's theorem and Doob's maximal inequality together with (2.6):

$$
\begin{gathered}
\mathbf{E}\left(\frac{1}{\delta} \int_{0}^{\delta} \max _{k \leqslant n}\left|\sum_{i=1}^{k} \hat{l}_{n, i}\left(\zeta_{n, i}^{s}(c)-\mathbf{E} \zeta_{n, i}^{s}(c)\right)\right| d c\right)^{2} \\
\leqslant \frac{1}{\delta} \int_{0}^{\delta} \mathbf{E} \max _{k \leqslant n}\left|\sum_{i=1}^{k} \hat{l}_{n, i}\left(\zeta_{n, i}^{s}(c)-\mathbf{E} \zeta_{n, i}^{s}(c)\right)\right|^{2} d c \\
\leqslant \frac{1}{\delta} \int_{0}^{\delta} \mathbf{E}\left(\sum_{i=1}^{n} \hat{l}_{n, i}\left(\zeta_{n, i}^{s}(c)-\mathbf{E} \zeta_{n, i}^{s}(c)\right)\right)^{2} d c
\end{gathered}
$$




$$
\begin{aligned}
& \leqslant \frac{1}{\delta} \int_{0}^{\delta} \sum_{i=1}^{n} \hat{l}_{n, i}^{2} \mathbf{E}\left(\zeta_{n, i}^{s}(c)-\mathbf{E} \zeta_{n, i}^{s}(c)\right)^{2} d c \\
& \leqslant s^{2} \sum_{i=1}^{n} \hat{l}_{n, i}^{2} \longrightarrow 0 \quad \text { as } n \rightarrow \infty .
\end{aligned}
$$

In order to study $\left(d_{6}\right)$, we show that

$$
\lim _{s \rightarrow \infty} \varlimsup_{n} \int_{0}^{\delta} \max _{k \leqslant n}\left|\sum_{i=1}^{k} \hat{l}_{n, i} \mathbf{E} \zeta_{n, i}^{s}(c)\right| d c=0
$$

Since

$$
\max _{k \leqslant n}\left|\sum_{i=1}^{k} \hat{l}_{n, i} \mathbf{E} \zeta_{n, i}^{s}(c)\right| \leqslant \sum_{i=1}^{n}\left|\hat{l}_{n, i}\right|\left|\mathbf{E} \zeta_{n, i}^{s}(c)\right|,
$$

let us see first that

$$
\sup _{i \leqslant n, c \in[0, \delta]}\left|\mathbf{E} \zeta_{n, i}^{s}(c)-\mathbf{E} \zeta_{n, i}^{s}(0)\right| \longrightarrow 0 \quad \text { as } \quad n \rightarrow \infty .
$$

Easy computations yield

$$
\sup _{i \leqslant n, c \in[0, \delta]}\left|\mathbf{E} \zeta_{n, i}^{s}(c)-\mathbf{E} \zeta_{n, i}^{s}(0)\right|=\sup _{i \leqslant n, c \in[0, \delta]} s \int\left|q\left(x-c l_{n, i}\right)-q(x)\right| d x
$$

ensuring (3.29) by continuity of translation in $L^{1}$ and integrability of $q$.

Next, since $\left(\zeta_{n, i}^{s}(0)\right)_{i \leqslant n}$ are identically distributed, we have by (2.7) that

$\sum_{i=1}^{n}\left|\hat{l}_{n, i}\right|\left|\mathbf{E} \zeta_{n, i}^{s}(c)\right| \leqslant \int_{0}^{1}\left|l^{\prime}(s)\right| d s\left(\left|\mathbf{E} \zeta_{n, 1}^{s}(0)\right|+\sup _{i \leqslant n, c \in[0, \delta]}\left|\mathbf{E} \zeta_{n, i}^{s}(c)-\mathbf{E} \zeta_{n, i}^{s}(0)\right|\right)$.

It follows that

$$
\begin{aligned}
& \int_{0}^{\delta} \max _{k \leqslant n}\left|\sum_{i=1}^{k} \hat{l}_{n, i} \mathbf{E} \zeta_{n, i}^{s}(c)\right| d c \\
& \quad \leqslant \delta \int_{0}^{1}\left|l^{\prime}(s)\right| d s\left(\left|\mathbf{E} \zeta_{n, 1}^{s}(0)\right|+\sup _{i \leqslant n, c \in[0, \delta]}\left|\mathbf{E} \zeta_{n, i}^{s}(c)-\mathbf{E} \zeta_{n, i}^{s}(0)\right|\right),
\end{aligned}
$$

now (3.29) and $\mathbf{E} \zeta_{n, 1}^{s}(0) \rightarrow 0$ as $s \rightarrow \infty$ (by dominated convergence) both ensure (3.28).

We start the study of $\left(d_{7}\right)$ with the following considerations:

$$
\max _{k \leqslant n}\left|\sum_{i=1}^{k} \hat{l}_{n, i} \zeta_{n, i}(c) \mathbf{1}_{\left|\zeta_{n, i}(c)\right|>s}\right| \leqslant \sum_{i=1}^{n}\left|\hat{l}_{n, i}\right|\left|\zeta_{n, i}(c)\right| \mathbf{1}_{\left|\zeta_{n, i}(c)\right|>s} .
$$

Since $\mathbf{E}\left|\zeta_{n, i}(c)\right| \mathbf{1}_{\left|\zeta_{n, i}(c)\right|>s}=\int|h(x)| \mathbf{1}_{|h(x)|>s} q\left(x-c l_{n, i}\right) d x$, we have

$$
\begin{aligned}
& \sup _{i \leqslant n, c \in[0, \delta]}|\mathbf{E}| \zeta_{n, i}(c)\left|\mathbf{1}_{\left|\zeta_{n, i}(c)\right|>s}-\mathbf{E}\right| \zeta_{n, i}(0)\left|\mathbf{1}_{\left|\zeta_{n, i}(0)\right|>s}\right| \\
& \quad \leqslant \int|h(x)| \mathbf{1}_{|h(x)|>s} \sup _{i \leqslant n, c \in[0, \delta]}\left|q\left(x-c l_{n, i}\right)-q(x)\right| d x .
\end{aligned}
$$


But obviously

and

$$
\sup _{i \leqslant n, c \in[0, \delta]}\left|q\left(x-c l_{n, i}\right)-q(x)\right| \longrightarrow 0 \quad \text { as } \quad n \rightarrow \infty
$$

$$
\begin{aligned}
& |h(x)| \mathbf{1}_{|h(x)|>s} \sup _{i \leqslant n, c \in[0, \delta]}\left|q\left(x-c l_{n, i}\right)-q(x)\right| \\
& \quad \leqslant|h(x)| q(x)+|h(x)| \mathbf{1}_{|x| \leqslant \delta h}+|h(x)| \mathbf{1}_{|x|>\delta h} q(|x|-\delta h) .
\end{aligned}
$$

The first two terms of the right-hand side are integrable, so is also the third one according to the following lemma proved in Appendix A.2.

Lemma 1. For all $\lambda>0$, we have $\int U^{\prime}(x) q(|x|-\lambda) d x<\infty$.

Thus dominated convergence ensures

$$
\sup _{i \leqslant n, c \in[0, \delta]}|\mathbf{E}| \zeta_{n, i}(c)\left|\mathbf{1}_{\left|\zeta_{n, i}(c)\right|>s}-\mathbf{E}\right| \zeta_{n, i}(0)\left|\mathbf{1}_{\left|\zeta_{n, i}(0)\right|>s}\right| \longrightarrow 0
$$

as $n \rightarrow \infty$. Then

$$
\begin{aligned}
& \mathbf{E} \int_{0}^{\delta} \max _{k \leqslant n}\left|\sum_{i=1}^{k} \hat{l}_{n, i} \zeta_{n, i}(c) \mathbf{1}_{\left|\zeta_{n, i}(c)\right|>s}\right|=\int_{0}^{\delta} \mathbf{E} \max _{k \leqslant n}\left|\sum_{i=1}^{k} \hat{l}_{n, i} \zeta_{n, i}(c) \mathbf{1}_{\left|\zeta_{n, i}(c)\right|>s}\right| d c \\
& \leqslant \sum_{i=1}^{n}\left|\hat{l}_{n, i}\right| \int_{0}^{\delta}|\mathbf{E}| \zeta_{n, i}(c)\left|\mathbf{1}_{\left|\zeta_{n, i}(c)\right|>s}-\mathbf{E}\right| \zeta_{n, i}(0)\left|\mathbf{1}_{\left|\zeta_{n, i}(0)\right|>s}\right| d c \\
& \quad+\delta \sum_{i=1}^{n}\left|\hat{l}_{n, i}\right| \mathbf{E}\left|\zeta_{1,1}(0)\right| \mathbf{1}_{\left|\zeta_{1,1}(0)\right|>s} .
\end{aligned}
$$

Since $\zeta_{1,1}(0)$ is a centered and integrable variable, dominated convergence ensures

$$
\mathbf{E}\left(\left|\zeta_{1,1}(0)\right| \mathbf{1}_{\left|\zeta_{1,1}(0)\right|>s}\right) \longrightarrow 0, \quad \mathbf{E} \zeta_{1,1}^{s}(0) \longrightarrow 0, \quad s \rightarrow \infty .
$$

Using (3.31) and (3.30), we obtain by (2.7)

$$
\lim _{s \rightarrow \infty} \varlimsup_{n} \mathbf{E} \int_{0}^{\delta} \max _{k \leqslant n}\left|\sum_{i=1}^{k} \hat{l}_{n, i} \zeta_{n, i}(c) \mathbf{1}_{\mid \zeta_{n, i}(c)>s}\right| d c=0 .
$$

Since

$$
\begin{aligned}
\frac{1}{\delta} \int_{0}^{\delta} & \left\|\sum_{i=1}^{[n \cdot]} \hat{l}_{n, i} \zeta_{n, i}(c)\right\| d c \leqslant \frac{1}{\delta} \int_{0}^{\delta}\left\|\sum_{i=1}^{[n \cdot]} \hat{l}_{n, i}\left(\zeta_{n, i}^{s}(c)-\mathbf{E} \zeta_{n, i}^{s}(c)\right)\right\| d c \\
+ & \frac{1}{\delta} \int_{0}^{\delta}\left\|\sum_{i=1}^{[n \cdot]} \hat{l}_{n, i} \zeta_{n, i}(c) \mathbf{1}_{\left|\zeta_{n, i}(c)\right|>s}\right\| d c+\frac{1}{\delta} \int_{0}^{\delta}\left\|\sum_{i=1}^{[n \cdot]} \hat{l}_{n, i} \mathbf{E} \zeta_{n, i}^{s}(c)\right\| d c
\end{aligned}
$$

using (3.27), (3.28), and (3.32) with the decomposition (3.26), we deduce $\mathbf{E}\left(\frac{1}{\delta} \int_{0}^{\delta}\left\|L_{n, S_{n}, c}-a l\right\| d c\right)=\mathbf{E}\left(\frac{1}{\delta} \int_{0}^{\delta}\left\|\sum_{i=1}^{[n \cdot]} \hat{l}_{n, i} \zeta_{n, i}(c)\right\| d c\right) \rightarrow 0, \quad n \rightarrow \infty$.

We obtain thus convergence (3.24) of $L_{n, S_{n}, c}$. 
It is easy to see also that we have for each fixed $c \in[0, \delta]$ the convergence of $L_{n, S_{n}, c}$ in probability to al:

$$
L_{n, S_{n}, c} \stackrel{\mathbf{P}}{\rightarrow} a l .
$$

Indeed, it suffices to prove the convergence in probability to 0 of (3.25). To this end we study the sums corresponding to $\left(d_{5}\right),\left(d_{6}\right)$, and $\left(d_{7}\right)$ as previously but without integrating in $c \in[0, \delta]$.

Since $G_{n, c} S_{n}(t)$ is absolutely continuous, one has

$$
G_{n, c} S_{n}(t)=S_{n}(t)+\int_{0}^{c} L_{n, S_{n}, s}(t) d s, \quad G_{\infty, c} W(t)=W(t)+c a l .
$$

It follows

$$
\begin{aligned}
\left\|G_{n, c} S_{n}-G_{\infty, c} W\right\| & \leqslant\left\|S_{n}-W\right\|+\sup _{t \in[0,1]}\left|\int_{0}^{c}\left(L_{n, S_{n}, s}(t)-a l(t)\right) d s\right| \\
& \leqslant\left\|S_{n}-W\right\|+\int_{0}^{\delta}\left\|L_{n, S_{n}, s}-a l\right\| d s
\end{aligned}
$$

and so

$$
\sup _{c \in[0, \delta]}\left\|G_{n, c} S_{n}-G_{\infty, c} W\right\| \stackrel{\mathbf{P}}{\longrightarrow} 0, \quad n \rightarrow \infty .
$$

\subsection{2 . Verification of the hypotheses of Proposition 5} for $\left(g_{n}\right)_{n \geqslant 1}$.

1. Absolute continuity of $g_{n}(\omega, \cdot), n \in \overline{\mathbf{N}}$. We use the fact that $f$ is locally Lipschitzian. The case of $g_{\infty}(\omega, \cdot)=h_{\infty}(\omega, \cdot)$ is obvious. To study $g_{n}(\omega, \cdot)=f\left(G_{n}, S_{n}\right)$, introduce $u^{(n)}: c \longmapsto\left(u_{1}^{(n)}(c), \ldots, u_{n}^{(n)}(c)\right)$, where

$$
u_{i}^{(n)}(c)=n^{-1 / 2} U\left(\eta_{i}^{n}+c l_{n, i}\right) .
$$

For all fixed $\omega \in \Omega$ and $\eta_{i}^{n}+c l_{n, i}$ remaining in a bounded domain, $u_{i}^{(n)}$ is absolutely continuous. We use the following simple fact.

Lemma 2. Let $\left(f_{1}, \ldots, f_{p}\right): \mathbf{R} \rightarrow V \subset \mathbf{R}^{p}$ be a mapping whose components are absolutely continuous and let $F: V \rightarrow \mathbf{R}$ be Lipschitzian. Then $G=F\left(f_{1}, \ldots, f_{p}\right)$ is absolutely continuous.

Let $\theta_{n}: \mathbf{R}^{n} \rightarrow \mathbb{E}$ be the following Lipschitzian function:

$$
\theta_{n}(x)(t)=\sum_{i=1}^{[n t]} x_{i}, \quad t \in[0,1]
$$

Note that

$$
\theta_{n}\left(u^{(n)}(c)\right)=G_{n, c} S_{n} .
$$

For fixed $\omega, n, c$ there is a convex open neighborhood $V\left(G_{n, c} S_{n}\right)$ of $G_{n, c} S_{n}$ on which $f$ is Lipschitzian. Since $G_{n, s} S_{n} \rightarrow G_{n, c} S_{n}$ for $s \rightarrow c$, there is $I_{c}(\omega, n)$, neighborhood of $c$ in $[0, \delta]$, such that for $s \in I_{c}(\omega, n)$,

$$
G_{n, s} S_{n}=\theta_{n}\left(u^{(n)}(s)\right) \in V\left(G_{n, c} S_{n}\right)
$$


Since $\theta_{n}$ is linear and $V\left(G_{n, c} S_{n}\right)$ is open and convex, we obtain $u^{(n)}(s) \in$ $\theta_{n}^{-1}\left(V\left(G_{n, c} S_{n}\right)\right)$. So

(a) $f \circ \theta_{n}$ is Lipschitzian on $\theta_{n}^{-1}\left(V\left(G_{n, c} S_{n}\right)\right)$;

(b) $u^{(n)}$ takes values in $\theta_{n}^{-1}\left(V\left(G_{n, c} S_{n}\right)\right)$ on $I_{c}(\omega, n)$ and has absolutely continuous components.

Lemma 2 ensures absolute continuity of $f \circ \theta_{n} \circ u^{(n)}=f\left(G_{n, \bullet} S_{n}\right)=$ $g_{n}(\omega, \cdot)$ on $I_{c}(\omega, n)$. Extracting a finite cover of $[0, \delta]$ by open sets $I_{c_{k}}(\omega, n)$, one obtains absolute continuity of $g_{n}(\omega, \cdot)$ for any $\omega, n$.

2. Point 3.6 (ii) asserts exactly that $g_{n}(\omega, 0) \stackrel{\mathbf{P}}{\longrightarrow} g_{\infty}(\omega, 0)$.

3. In order to see $g_{n}(\omega, \delta) \stackrel{\mathbf{P}}{\longrightarrow} g_{\infty}(\omega, \delta)$, we use point (i) of Theorem B already checked and Skorokhod's representation theorem to derive

$$
G_{n, \delta} S_{n} \stackrel{\mathbf{P}}{\longrightarrow} G_{\infty, \delta} W, \quad n \rightarrow \infty .
$$

We conclude this step using the continuity of $f$ at $W+\delta a l \in B\left(x, 2 r_{3}\right)$ for $\omega \in W^{-1}(V)$.

4. We study here $\frac{\partial}{\partial c} g_{n}(\omega, c), n \in \overline{\mathbf{N}}$.

From the absolute continuity proved in point 1 we deduce that for all fixed $\omega, n$, the derivatives $\frac{\partial}{\partial c} g_{n}(\omega, c), \frac{\partial}{\partial c} g_{\infty}(\omega, c)$ are defined on a set $A(\omega) \in$ $\mathscr{B}([0, \delta]), \lambda(A(\omega))^{\complement}=0$. We know already from 3.6 that for all $\omega \in W^{-1}(V)$ almost all $c$

$$
\frac{\partial}{\partial c} g_{\infty}(\omega, c)=D_{a l} f\left(G_{\infty, c} W\right) .
$$

For fixed $\omega, n$, we show that $f$ is differentiable in the tangent direction $L_{n, S_{n}, c}$ at $G_{n, c} S_{n}$ and on a set of measure 1

$$
\frac{\partial}{\partial c} g_{n}(\omega, c)=D_{L_{n, S_{n}, c}} f\left(G_{n, c} S_{n}\right)
$$

Indeed since for almost all $c$,

$$
\frac{f\left(G_{n, c+h} S_{n}\right)-f\left(G_{n, c} S_{n}\right)}{h} \longrightarrow \frac{\partial}{\partial c} g_{n}(\omega, c), \quad h \rightarrow 0,
$$

to prove (3.35) it suffices to establish that

$$
\lim _{h \rightarrow 0}\left\|\frac{f\left(G_{n, c} S_{n}+h L_{n, S_{n}, c}\right)-f\left(G_{n, c} S_{n}\right)}{h}-\frac{f\left(G_{n, c+h} S_{n}\right)-f\left(G_{n, c} S_{n}\right)}{h}\right\|=0 .
$$

The latter identity follows from the fact that $f$ is locally Lipschitzian and from the almost sure existence of $L_{n, S_{n}, c}$ giving

$$
\frac{G_{n, c} S_{n}+h L_{n, S_{n}, c}-G_{n, c+h} S_{n}}{h} \longrightarrow 0, \quad h \rightarrow 0 .
$$

5. Since $g_{\infty}=h_{\infty}$, the bound $\frac{\partial}{\partial c} g_{\infty}(\omega, c)>0$ a.s. for $\omega \in W^{-1}(V)$ follows from 3.6 (iv). 
6. Verification of hypothesis 4) of Proposition 5 for $\left(g_{n}\right)$. The purpose is to finish checking point 4$)$ for $\left(g_{n}\right)$ by proving that

$$
\left\|\frac{\partial}{\partial c} g_{n}(\omega, \cdot)-\frac{\partial}{\partial c} g_{\infty}(\omega, \cdot)\right\|_{L^{1}([0, \delta])} \stackrel{\mathbf{P}}{\longrightarrow} 0, \quad n \rightarrow \infty .
$$

Let $\left(n^{\prime}\right)$ be any subsequence; we begin with extracting a further subsequence $\left(n^{\prime \prime}\right) \subset\left(n^{\prime}\right)$ with almost sure convergences (3.24), (3.33), (3.34). With $\omega$ in this almost sure set, we have seen that for $\bar{\lambda}$-almost all $c \in[0, \delta]$

$$
\frac{\partial}{\partial c} g_{n^{\prime \prime}}(\omega, c)=D_{L_{n^{\prime \prime}, s_{n^{\prime \prime}}, c}} f\left(G_{n^{\prime \prime}, c} S_{n^{\prime \prime}}\right), \quad \frac{\partial}{\partial c} g_{\infty}(\omega, c)=D_{a l} f\left(G_{\infty, c} W\right) .
$$

There is $N(\omega)$ such that for $n^{\prime \prime} \geqslant N(\omega)$ and $c \in[0, \delta], G_{n^{\prime \prime}, c} S_{n^{\prime \prime}} \in B\left(x, r_{2}\right)$. Then

$$
\begin{aligned}
& \left\|\frac{\partial}{\partial c} g_{n^{\prime \prime}}(\omega, \cdot)-\frac{\partial}{\partial c} g_{\infty}(\omega, \cdot)\right\|_{L^{1}([0, \delta])} \\
& =\int_{[0, \delta]}\left|D_{L_{n^{\prime \prime}, S_{n^{\prime \prime}, c}}} f\left(G_{n^{\prime \prime}, c} S_{n^{\prime \prime}}\right)-D_{a l} f\left(G_{\infty, c} W\right)\right| d c \\
& \leqslant \int_{[0, \delta]}\left|D_{L_{n^{\prime \prime}, S_{n^{\prime \prime}, c}}\left\|L_{n^{\prime \prime}, s_{n^{\prime \prime}}, c}\right\|^{-1}} f\left(G_{n^{\prime \prime}, c} S_{n^{\prime \prime}}\right)\right|\left|\left\|L_{n^{\prime \prime}, S_{n^{\prime \prime}, c}}\right\|-a\|l\|\right| d c \\
& \quad+a\|l\| \int_{[0, \delta]}\left|D_{L_{n^{\prime \prime}, S_{n^{\prime \prime}}, c}\left\|L_{n^{\prime \prime}, S_{n^{\prime \prime}, c}}\right\|^{-1}} f\left(G_{n^{\prime \prime}, c} S_{n^{\prime \prime}}\right)-D_{l /\|l\|} f\left(G_{\infty, c} W\right)\right| d c .
\end{aligned}
$$

Since $\left(G_{n^{\prime \prime}, c} S_{n^{\prime \prime}}, L_{n^{\prime \prime}, S_{n^{\prime \prime}, c}}\left\|L_{n^{\prime \prime}, S_{n^{\prime \prime}, c}}\right\|^{-1}\right) \in A$ and $\left(G_{\infty, c} W, l /\|l\|\right) \in A$, where $A$ is a set on which $D \cdot f(\cdot)$ is bounded, the first term on the right-hand side of the later inequality goes to zero because of (3.24). Since, moreover,

$$
G_{n^{\prime \prime}, c} S_{n^{\prime \prime}} \longrightarrow G_{\infty, c} W, \quad \frac{L_{n^{\prime \prime}, S_{n^{\prime \prime}, c}}}{\left\|L_{n^{\prime \prime}, S_{n^{\prime \prime}, c}}\right\|} \longrightarrow \frac{l}{\|l\|},
$$

we have

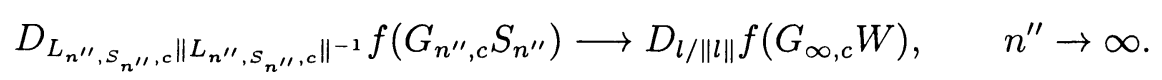

Since the sequence is bounded, by dominated convergence, the second term also goes to 0. Finally, we obtain (3.36) and point 4) of Proposition 5 for $\left(g_{n}\right)_{n \geqslant 1}$.

3.7.3. Conclusion for sequence $\left(g_{n}\right)_{n \geqslant 1}$. Applying Proposition 5 yields: for any $s>0$,

$$
\lim _{n \rightarrow \infty} \mathbf{P}\left\{\omega \in W^{-1}(V) \mid\left\|\lambda_{[0, \delta]} g_{n}(\omega, \cdot)^{-1}-\lambda_{[0, \delta]} g_{\infty}(\omega, \cdot)^{-1}\right\|>s\right\}=0 .
$$

3.8. Final verification of (iv) of Theorem B. First, since $h_{\infty}=g_{\infty}$, we obtain

$$
\begin{aligned}
& \left\|\lambda_{[0, \delta]} g_{n}(\omega, \cdot)^{-1}-\lambda_{[0, \delta]} h_{n}(\omega, \cdot)^{-1}\right\| \leqslant\left\|\lambda_{[0, \delta]} g_{n}(\omega, \cdot)^{-1}-\lambda_{[0, \delta]} g_{\infty}(\omega, \cdot)^{-1}\right\| \\
& +\left\|\lambda_{[0, \delta]} h_{n}(\omega, \cdot)^{-1}-\lambda_{[0, \delta]} h_{\infty}(\omega, \cdot)^{-1}\right\| .
\end{aligned}
$$


We deduce from the studies of $h_{n}, g_{n}$ leading to (3.21), (3.37), that for any $\alpha>0$

$$
\lim _{n \rightarrow \infty} \mathbf{P}\left\{\omega \in W^{-1}(V) \mid\left\|\lambda_{[0, \delta]} g_{n}(\omega, \cdot)^{-1}-\lambda_{[0, \delta]} h_{n}(\omega, \cdot)^{-1}\right\|>\alpha\right\}=0 .
$$

Since $\lambda_{[0, \delta]} g_{n}(\omega, \cdot)^{-1}=\lambda_{[0, \delta]} \varphi_{n, S_{n}}^{-1}$ and $\lambda_{[0, \delta]} h_{n}(\omega, \cdot)^{-1}=\lambda_{[0, \delta]} \varphi_{\infty, S_{n}}^{-1}$, one has

$$
\begin{aligned}
\mathbf{P} & \{\omega \\
& \left.\in S_{n}^{-1}(V) \mid\left\|\lambda_{[0, \delta]} \varphi_{n, S_{n}}^{-1}-\lambda_{[0, \delta]} \varphi_{\infty, S_{n}}^{-1}\right\|>\alpha\right\} \\
& \mathbf{P}\left\{\omega \in W^{-1}(V) \mid\left\|\lambda_{[0, \delta]} \varphi_{n, S_{n}}^{-1}-\lambda_{[0, \delta]} \varphi_{\infty, S_{n}}^{-1}\right\|>\alpha\right\} \\
& +\mathbf{P}\left(S_{n}^{-1}(V) \backslash W^{-1}(V)\right) .
\end{aligned}
$$

Since $S_{n} \rightarrow W$ and $P(\partial V)=0$, we get $\mathbf{P}\left(S_{n} \in V \backslash W \in V\right) \rightarrow 0$ and it follows that

$$
\lim _{n \rightarrow \infty} P_{n}\left\{x \in V \mid\left\|\lambda_{[0, \delta]} \varphi_{n, x}^{-1}-\lambda_{[0, \delta]} \varphi_{\infty, x}^{-1}\right\|>\alpha\right\}=0 .
$$

Denoting $A_{n, \alpha}=\left\{x \mid\left\|\lambda_{[0, \delta]} \varphi_{n, x}^{-1}-\lambda_{[0, \delta]} \varphi_{\infty, x}^{-1}\right\|>\alpha\right\}$, we have

$$
\begin{aligned}
\int_{V} & \left\|\lambda_{[0, \delta]} \varphi_{n, x}^{-1}-\lambda_{[0, \delta]} \varphi_{\infty, x}^{-1}\right\| P_{n}(d x) \\
= & \int_{V \cap A_{n, \alpha}}\left\|\lambda_{[0, \delta]} \varphi_{n, x}^{-1}-\lambda_{[0, \delta]} \varphi_{\infty, x}^{-1}\right\| P_{n}(d x) \\
& +\int_{V \cap A_{n, \alpha}^{c}}\left\|\lambda_{[0, \delta]} \varphi_{n, x}^{-1}-\lambda_{[0, \delta]} \varphi_{\infty, x}^{-1}\right\| P_{n}(d x) \\
& \leqslant 2 P_{n}\left\{x \in V \mid\left\|\lambda_{[0, \delta]} \varphi_{n, x}^{-1}-\lambda_{[0, \delta]} \varphi_{\infty, x}^{-1}\right\|>\alpha\right\}+\alpha \int_{V \cap A_{n, \alpha}^{c}} P_{n}(d x) .
\end{aligned}
$$

Using first (3.38) as $n \rightarrow \infty$ and then letting $\alpha \rightarrow 0$, we obtain finally point (iv) of Theorem B.

3.9. Conclusion. Finally applying Theorem B, we prove Theorem 1 and obtain (1.3).

\section{Examples of functionals in $\mathscr{M}_{P}^{(1)}$}

We prove in this section Proposition 1 and 2.

First, the property stated in Proposition 2 is clear since conditions of Theorem 19.1 in [4] are satisfied and ensure that $\mathscr{M}_{P}^{(1)}$ is a proper subset of $\mathscr{M}_{P}^{(a)}$ for which the property is known (see [4, Section 19]).

We discuss now examples cited in Proposition 1.

\subsection{Study of sup type functional.}

4.1.1. Differentiability. Consider $g$ defined by (1.4) and let $S_{0}=$ $\left\{t \in \mathbf{R} \mid \varphi^{\prime}(t) \neq 0\right\} \in \mathscr{B}(\mathbf{R}), \lambda\left(S_{0}^{c}\right)=0$. Let $M_{x}=\{t \in[0,1] \mid g(x)=$ $\varphi(x(t))\}$; since $\varphi$ is convex, card $M_{x} \in\{1,2\}$. 
R e m a r k 4. Moreover, since vector $\left(\sup _{t \in[0,1]} W(t), \inf _{t \in[0,1]} W(t)\right)$ has a density (see $[1$, Section 11]), we have even for $P$-almost all $x$, card $M_{x}=1$.

Using [8], we can define $t_{x}=\operatorname{argmax}_{t \in[0,1]} \varphi(x(t)), P$-a.s. We can derive from absolute continuity of $\sup _{t \in[0,1]} W(t)$ and $\inf _{t \in[0,1]} W(t)$ that $x\left(t_{x}\right) \in S_{0}$, $P$-a.s.

We have the following differentiability property.

Lemma 3. For all $x, l \in C([0,1]), g$ is differentiable at $x$ in the direction l:

$$
D_{l}^{-} g(x)=\inf _{t \in M_{x}} \varphi^{\prime}(x(t)) l(t), \quad D_{l}^{+} g(x)=\max _{t \in M_{x}} \varphi^{\prime}(x(t)) l(t),
$$

where $D_{l}^{-}, D_{l}^{+}$mean left and right weak derivatives along $l$.

P r o o f. Using convexity of $\varphi$, we have for $t_{x} \in M_{x}$ and $t_{c} \in M_{x+c l}$ :

$$
\varphi^{\prime}\left(x\left(t_{x}\right)\right) c l\left(t_{x}\right) \leqslant g(x+c l)-g(x) \leqslant \varphi^{\prime}\left(x\left(t_{c}\right)+c l\left(t_{c}\right)\right) c l\left(t_{c}\right) .
$$

It is easy to observe that for any positive sequence $\left(c_{n}\right)_{n \geqslant 1}$ going to 0 and $t_{n} \in M_{x+c_{n} l}$, we can extract $\left(c_{n^{\prime}}\right)$ such that $t_{n^{\prime}} \rightarrow t_{0} \in M_{x}, n^{\prime} \rightarrow \infty$.

Let $\left(c_{n^{\prime}}\right)$ be extracted from any $\left(c_{n}\right)_{n \geqslant 1}$ positive going to zero; we can extract further $\left(c_{n^{\prime \prime}}\right)$ with $t_{n^{\prime \prime}} \rightarrow t_{0} \in M_{x}$. Since $\varphi^{\prime}$ is continuous, we derive from (4.2) for any $t_{x} \in M_{x}$ :

$$
\begin{aligned}
\varphi^{\prime}\left(x\left(t_{x}\right)\right) l\left(t_{x}\right) & \leqslant \lim _{n^{\prime \prime} \rightarrow \infty} \frac{g\left(x+c_{n^{\prime \prime}} l\right)-g(x)}{c} \\
& \leqslant \varlimsup_{n^{\prime \prime} \rightarrow \infty} \frac{g\left(x+c_{n^{\prime \prime}} l\right)-g(x)}{c} \leqslant \varphi^{\prime}\left(x\left(t_{0}\right)\right) l\left(t_{0}\right) .
\end{aligned}
$$

Taking maximum over $t_{x} \in M_{x}$, we deduce that from any $\left(c_{n^{\prime}}\right)$, we can extract $\left(c_{n^{\prime \prime}}\right)$ with the same relative limit

$$
\lim _{n \rightarrow \infty} \frac{g\left(x+c_{n^{\prime \prime}} l\right)-g(x)}{c}=\max _{t \in M_{x}} \varphi^{\prime}(x(t)) l(t) .
$$

So the second part of (4.1) holds; similar computations yield its first part. Lemma 3 is proved.

\subsubsection{Verification of the conditions for a functional to belong} to $\mathscr{M}_{P}^{(1)}$. Let $x \in B_{0}$, where $B_{0}$ is the measurable set for which $t_{x}=$ $\operatorname{argmax}_{t \in[0,1]} \varphi(x(t))$ is well defined (see Remark 4); in this case $D_{l} g(x)=$ $\varphi^{\prime}\left(x\left(t_{x}\right)\right) l\left(t_{x}\right)$. Consider the neighborhood $B(x, 1)$ of $x$.

1. With $M=\|x\|+1$, since $\varphi$ is Lipschitzian on $[-M, M]$ with some positive constant $K_{M}$, we easily obtain

$$
|g(y)-g(x)| \leqslant \sup _{t \in[0,1]}|\varphi(x(t))-\varphi(y(t))| \leqslant K_{M}\|x-y\| .
$$

2. Since $t_{x}$ realizes $\sup _{t \in[0,1]} \varphi(x(t))$, convexity of $\varphi$ ensures $\varphi^{\prime}(x(t)) \neq 0$. We thus easily find $l \in H_{P}$ with $D_{l} g(x) \neq 0$. 
3. Recall $A=\left\{(y, l) \mid\|l\|=1, D_{l} g(y)\right.$ defined $\}$.

For $(y, l) \in A, D_{l} g(y)$ exists; thanks to Lemma 3 , we have

$$
D_{l} g(y)=\varphi^{\prime}\left(y\left(t_{y}\right)\right) l\left(t_{y}\right), \quad \text { for } \quad t_{y} \in M_{y} .
$$

Since $y \in B(x, 1)$, we have that $D_{l} g(y)$ is bounded by $K_{M}\|l\|$.

For $\left(y_{n}, l_{n}\right) \rightarrow(y, l) \in A$, since derivatives exist, we have, for $t_{n} \in M_{y_{n}}$,

$$
D_{l_{n}} g\left(y_{n}\right)=\varphi^{\prime}\left(y_{n}\left(t_{n}\right)\right) l_{n}\left(t_{n}\right) .
$$

For any $\left(n^{\prime}\right)$, we can extract $\left(n^{\prime \prime}\right)$ with $t_{n^{\prime \prime}} \rightarrow t_{0} \in M_{y}$ and obtain as $n^{\prime \prime} \rightarrow \infty$

$$
\varphi^{\prime}\left(y_{n^{\prime \prime}}\left(t_{n^{\prime \prime}}\right)\right) l_{n^{\prime \prime}}\left(t_{n^{\prime \prime}}\right) \longrightarrow \varphi^{\prime}\left(y\left(t_{0}\right)\right) l\left(t_{0}\right)=D_{l} g(y) .
$$

Since the limit does not depend on subsequence $\left(n^{\prime}\right)$, we have the required continuity.

$\mathrm{R}$ e $\mathrm{m}$ a r k 5. As mentioned in Remark 1, we can weaken the conditions on $\varphi$ in (1.4). In this case, Lemma 3 does not hold anymore, but we can exhibit $B_{0} \in \mathscr{B}(\mathbf{R})$ with $\lambda\left(B_{0}^{c}\right)=0$ such that for $x \in B_{0}$ and $l \in C([0,1])$, $D_{l} g(x)=\varphi^{\prime}\left(x\left(t_{x}\right)\right) l\left(t_{x}\right), t_{x} \in M_{x}$. The point 3 must be revised in such a way that failing of being in $\mathscr{M}_{P}^{(1)}$, at least the proof of Theorem 1 still works and conclusion (1.3) remains valid.

4.2. Study of integral type functional. We consider the functional given by (1.5), namely

$$
h(x)=\int_{0}^{1} q(x(t)) d t
$$

where $q$ is $C^{1}$ and such that $q^{\prime}$ does not vanish almost everywhere in some interval $(-\alpha, \alpha)$, for $\alpha>0$. We suppose moreover $(-\alpha, \alpha)$ is the biggest such interval.

4.2.1. Differentiability. For $B_{0}=\left\{x \in([0,1]) \mid \lambda\left\{t \mid x(t) \in S_{0}\right\}=1\right\}$, we have $P\left(B_{0}\right)=1$.

Lemma 4. For $x, l \in C([0,1])$, we have

$$
D_{l} h(x)=\int_{0}^{1} q^{\prime}(x(t)) l(t) d t
$$

$\mathrm{P} r$ o o f. It is not difficult to apply the dominated convergence theorem to obtain

$$
\begin{aligned}
& \frac{h(x+c l)-h(x)}{c}-\int_{0}^{1} q^{\prime}(x(t)) l(t) d t \\
& \quad=\int_{0}^{1} \frac{q(x(t)+c l(t))-q(x(t))}{c}-l(t) q^{\prime}(x(t)) d t
\end{aligned}
$$

which goes to zero as $c \rightarrow 0$. Lemma 4 is proved. 
4.2.2. Verification of the conditions for a functional to belong to $\mathscr{M}_{P}^{(1)}$. Let $x$ be such that $x(0)=0$ and consider the neighborhood $B(x, 1)$ of $x$.

1. Let $M=\|x\|+1$; we have

$|h(x)-h(y)| \leqslant \int_{0}^{1}|q(x(t))-q(y(t))| d t \leqslant K_{M} \int_{0}^{1}|x(t)-y(t)| d t \leqslant K_{M}\|x-y\|$.

2. The derivative is given in (4.3); moreover, there is $a>0$ such that for $0 \leqslant t<a, x(t) \in(-\alpha, \alpha)$ a.e., $q^{\prime}(x(t)) \neq 0$ a.e. We thus find some $l \in C([0,1])$ with $D_{l} h(x) \neq 0$. The space $H_{P}$ is dense in $C([0,1])$, this allows one to derive $l \in H_{P}$ with $D_{l} h(x) \neq 0$.

3. We have first $\left\|D_{l} h(y)\right\| \leqslant K_{M}$ for $(y, l) \in A$. And if $\left(y_{n}, l_{n}\right) \rightarrow(y, l)$, we have

$$
\begin{aligned}
\left|D_{l_{n}} h\left(y_{n}\right)-D_{l} h(y)\right| \leqslant & \int_{0}^{1}\left|q^{\prime}\left(y_{n}(t)\right) l_{n}(t)-q^{\prime}(y(t)) l_{n}(t)\right| d t \\
& +\int_{0}^{1}\left|q^{\prime}(y(t)) l_{n}(t)-q^{\prime}(y(t)) l(t)\right| d t \\
\leqslant & (\|l\|+1) \int\left|q^{\prime}\left(y_{n}(t)\right)-q^{\prime}(y(t))\right| d t+K_{M}\left\|l_{n}-l\right\| .
\end{aligned}
$$

Since dominated convergence implies that the first term of the right-hand side of the later inequality goes to zero, we obtain the required continuity.

$\mathrm{R} \mathrm{e} \mathrm{m} \mathrm{a} \mathrm{r} \mathrm{k} \mathrm{6.} \mathrm{Once} \mathrm{more,} \mathrm{we} \mathrm{can} \mathrm{weaken} \mathrm{the} \mathrm{conditions} \mathrm{of} \mathrm{Proposi-}$ tion 1 for functionals (1.5) assuming $q$ is Lipschitzian on all compacts with $q^{\prime}$ almost everywhere not zero. But as previously, we can check only conditions such that the proof of Theorem 1 still works and conclusion (1.3) remains anyway valid. But we cannot any longer guarantee $h \in \mathscr{M}_{P}^{(1)}$.

\section{A. Appendices}

\section{A.1. Asymptotic estimation for $U$.}

Proposition 6. Let $\xi$ be a random variable in $L^{p}(\Omega, \mathscr{F}, \mathbf{P})$. Denoting $F$ its distribution, we have the following estimate for $U=F^{-1} \circ \Phi$ :

$$
U(x)=o\left(\left(\frac{|x|}{q(x)}\right)^{1 / p}\right) \quad \text { at } \quad \pm \infty
$$

where $\Phi$ and $q$ denotes respectively distribution and density of $\mathscr{N}(0,1)$.

$\mathrm{Pr}$ o of. We use the following elementary result.

Lemma 5. Let $g:[0,1] \rightarrow \mathbf{R}^{+}$be nonincreasing and such that $\int_{0}^{1} g(t) d t<\infty$; then $g(t)=o(1 / t)$ as $t \rightarrow 0$.

First $\mathbf{E} \xi_{1}^{p}=\int_{0}^{1} F^{-1}(t)^{p} d t<\infty$. Lemma 5 ensures $F^{-1}(t)=o(1 /(1-$ $\left.t)^{1 / p}\right)$. Since $U(x)=F^{-1} \circ \Phi(x), \Phi(x) \rightarrow 1$ as $x \rightarrow \infty$ and $1-\Phi(x) \sim q(x) / x$ at $+\infty$, we obtain (A.1) for $x \rightarrow+\infty$. Similar arguments yield also (A.1) for $x \rightarrow-\infty$. Proposition 6 is proved. 
A.2. Technical lemma. Remind $q$ stands for the $\mathscr{N}(0,1)$ 's density. We justify Lemma 1 useful for $\left(d_{7}\right)$. We have to show that, for any $\lambda>0$,

$$
\int U^{\prime}(x) q(|x|-\lambda) d x<\infty .
$$

To do this we split the integral in the following way:

$$
\int U^{\prime}(x) q(|x|-\lambda) d x=\int_{|x| \leqslant|\lambda|}+\int_{|x|>|\lambda|} .
$$

We have

$$
\begin{aligned}
& \int_{|x| \leqslant|\lambda|} U^{\prime}(x) q(|x|-\lambda) d x \leqslant \int_{|x| \leqslant|\lambda|} U^{\prime}(x) d x \leqslant U(\lambda)-U(-\lambda)<\infty, \\
& \int_{|x|>|\lambda|} U^{\prime}(x) q(|x|-\lambda) d x \leqslant \int_{|x|>|\lambda|} q(|x|-|\lambda|) d U(x) .
\end{aligned}
$$

Since by (A.1): $U(n+1)=o\left(\sqrt{(n+1) / e^{-(n+1)^{2} / 2}}\right)$, comparing integral to a series, we obtain

$$
\int_{|x|>|\lambda|} q(|x|-|\lambda|) d U(x) \leqslant \sum_{n \geqslant|\lambda|} q(n-|\lambda|) U(n+1)<\infty .
$$

Lemma 1 is proved.

A.3. Key proposition. We prove Proposition 4 using Proposition 3 about convergence in variation of image measure.

For any subsequence $\left(n^{\prime}\right)$, we intend to show that there exists $\left(n^{\prime \prime}\right) \subset\left(n^{\prime}\right)$ such that

$$
\delta_{n^{\prime \prime}}(\omega)=\left\|\lambda_{[0, \delta]} f_{n^{\prime \prime}}(\omega, \cdot)^{-1}-\lambda_{[0, \delta]} f_{\infty}(\omega, \cdot)^{-1}\right\| \longrightarrow 0 \quad \text { as } \quad n^{\prime \prime} \rightarrow \infty
$$

for almost all $\omega \in \Omega^{*}$; as soon as (A.2) is proved, classical criteria of convergence in probability will ensure

$$
\forall \alpha>0, \quad \lim _{n \rightarrow \infty} \mathbf{P}\left\{\omega \in \Omega^{*} \mid\left\|\lambda_{[0, \delta]} f_{n}(\omega, \cdot)^{-1}-\lambda_{[0, \delta]} f_{\infty}(\omega, \cdot)^{-1}\right\|>\alpha\right\}=0 .
$$

Hypotheses 2, 3, 5 ensure there is a subsequence $\left(n^{\prime \prime}\right) \subset\left(n^{\prime}\right)$ with

$$
\begin{aligned}
& f_{n^{\prime \prime}}(\omega, 0) \longrightarrow f_{\infty}(\omega, 0) \quad \text { for all } \quad \omega \in \Omega_{2}^{*}, \quad \mathbf{P}\left(\Omega_{2}^{*}\right)=\mathbf{P}\left(\Omega^{*}\right) ; \\
& f_{n^{\prime \prime}}(\omega, \delta) \longrightarrow f_{\infty}(\omega, \delta) \quad \text { for all } \omega \in \Omega_{3}^{*}, \quad \mathbf{P}\left(\Omega_{3}^{*}\right)=\mathbf{P}\left(\Omega^{*}\right) ; \\
& \frac{\partial}{\partial c} f_{n^{\prime \prime}}(\omega, c) \longrightarrow \frac{\partial}{\partial c} f_{\infty}(\omega, c) \quad \text { for all } \quad(\omega, c) \in E_{5}^{*} \subset \Omega^{*} \otimes[0, \delta], \\
& P \otimes \bar{\lambda}\left(E_{5}^{*}\right)=P\left(\Omega^{*}\right) .
\end{aligned}
$$

Let $\Omega_{4}^{*} \subset \Omega^{*}, \mathbf{P}\left(\Omega_{4}^{*}\right)=\mathbf{P}\left(\Omega^{*}\right)$, be such that for all $\omega \in \Omega_{4}^{*}$, and all $n \geqslant N_{4}(\omega)$,

$$
\frac{\partial}{\partial c} f_{n^{\prime \prime}}(\omega, c)>0, \quad \frac{\partial}{\partial c} f_{\infty}(\omega, c)>0 \quad \bar{\lambda} \text {-a.e. }
$$


Fubini's theorem yields that there is $\Omega_{5}^{*} \subset \Omega^{*}, \mathbf{P}\left(\Omega_{5}^{*}\right)=\mathbf{P}\left(\Omega^{*}\right)$ such that

$$
\forall \omega \in \Omega_{5}^{*}, \quad \frac{\partial}{\partial c} f_{n^{\prime \prime}}(\omega, c) \longrightarrow \frac{\partial}{\partial c} f_{\infty}(\omega, c) \quad \bar{\lambda} \text {-a.e. for } \quad c \in[0, \delta] .
$$

Let consider

$$
\Omega_{0}^{*}=\Omega_{2}^{*} \cap \Omega_{3}^{*} \cap \Omega_{4}^{*} \cap \Omega_{5}^{*} \subset \Omega^{*}, \quad \mathbf{P}\left(\Omega_{0}^{*}\right)=\mathbf{P}\left(\Omega^{*}\right) .
$$

For all $\omega \in \Omega_{0}^{*}$ and $n \geqslant \max \left(N_{1}(\omega), N_{4}(\omega)\right)$, the functional $c \longmapsto f_{n^{\prime \prime}}(\omega, c)$ satisfies hypotheses of Proposition 3, and (A.2) readily follows.

Proposition 5 can be proved from Corollary 1 of [4] in the same way.

Acknowledgments. The authors would like to thank R. Zitikis for his careful reading of the paper and the anonymous referee for his valuable comments. The first author thanks also the Laboratoire de Statistique et Probabilités of the University Lille 1, where this work has been done.

\section{REFERENCES}

1. Биллингсли П. Сходимость вероятностных мер. М.: Наука, 1977, 352 с.

2. Давыљов Ю.А., Лифиич М.А. Метод расслоений в некоторых вероятностных задачах. - Итоги науки и техники, сер. теория вероятн., матем. статист., теорет. кибернет., 1984, т. 22, с. 61-158.

3. Давыдов Ю. А. О сходимости по вариации образов одномерных мер. - Записки научн. сем. ПОМИ, 1992 , т. 194, с. $48-58$.

4. Давыдов Ю. А., Лифиич М. А., Смородина Н. В. Локальные свойства распределений стохастических функционалов. М.Наука, 1995, 255 с.

5. Erdös P., Kac M. On certain limit theorem in the theory of probability. - Bull. Amer. Math. Soc., 1946, v. 52, p. 292-302.

6. Ибрагимов И.А., Линник Ю.В. Независимые и стационарно связанные величины. М.: Наука, 1965, 524 с.

7. Karatzas I., Shreve S.E. Brownian Motion and Stochastic Calculus. New York: Springer-Verlag, 1988, $470 \mathrm{p}$.

8. Цирельсон Б. С. Плотность распределения максимума гауссовского процесса. Теория вероятн. и ее примен., 1975 , т. 20 , в. 4 , с. $865-873$.

Поступила в редакцию

9.VII.2002

Исправленный вариант

30.X.2003 